\title{
Crisis y recuperación económica en los estados de la frontera norte. Un análisis de los ciclos económicos
}

\author{
Eliseo Díaz González, ${ }^{*}$ Mario Alberto Mendoza Sánchez ${ }^{* *}$
}

\section{Resumen}

Este trabajo analiza la perspectiva de recuperación económica y el comovimiento de la economía regional y nacional con un enfoque de ciclos económicos en 1997-2010. Se estiman la descomposición de tendencia y ciclo de crecimiento en cada entidad con el filtro Hodrick-Prescott y un modelo autorregresivo, utilizando datos de empleo. Las evidencias revelan que Nuevo León tiene capacidad para regresar a su tendencia de crecimiento, pero Baja California y Chihuahua tienen menor posibilidad. Finalmente, la dinámica de corto plazo de estas economías muestra que el grado de sincronización con la economía nacional parece jugar a favor de la recuperación del crecimiento.

Palabras clave: ciclos económicos, recuperación económica, frontera norte.

\begin{abstract}
This paper analyzes the prospect of economic recovery and the comovement of regional and national economy with a focus on business cycles theory in 1997-2010. We estimate the trend and cycle of composition of growth in each entity with the Hodrick-Prescott filter and an autoregressive model, using employment data. The evidence shows that Nuevo Leon has the capacity to return to its trend growth, but Baja California and Chihuahua has less possibility. Finally, short-term dynamics of these economies shows that the degree of synchronization with the national economy seems to play for the recovery of growth.
\end{abstract}

Keywords: business cycles, economic recuperation, northern border.

* Profesor-investigador del Departamento de Estudios Económicos, El Colegio de la Frontera Norte. Correo electrónico: ediaz@colef.mx.

** Profesor de la Universidad de Sonora, Hermosillo. Correo electrónico: mamendoza@pitic.uson.mx. 


\section{Introducción}

El análisis económico regional o de las economías estatales usualmente está restringido por diversas dificultades: las series de tiempo de las variables tienen un rezago de uno o dos años; las variables macroeconómicas fundamentales están disponibles sólo en una base anual y en comparación con las estadísticas nacionales, la contabilidad económica de los estados involucra sólo un número limitado de éstas. La suma de estas restricciones se expresa en forma más acentuada en las dificultades para hacer alguna predicción medianamente sustentada sobre el comportamiento económico que pueda seguir alguna entidad federativa.

La teoría de los ciclos económicos reales es una herramienta poderosa para predecir la reacción que una economía puede tener frente a shocks o innovaciones exógenas, que es una condición para describir alguna línea de base o la trayectoria previsible que pueda seguir la actividad económica en un espacio económico cualquiera, sea nacional o subnacional. Con este dato podemos aproximarnos a predecir cierto comportamiento económico general de los estados, aunque sin la precisión que debiera lograrse con un modelo macroeconómico consistente.

De igual modo, es posible conocer las características dinámicas de las economías regionales que explican su capacidad para pronunciar sus etapas de expansión y concretar una política regional ${ }^{1}$ con más incidencia sobre las actividades productivas que permitan a la población contar con elementos materiales para su reproducción y bienestar. Por último, el momento histórico por el que atraviesa el sistema económico -expresado en la pasada recesión de la economía internacional y nacional- da lugar al resurgimiento de la teoría de ciclos y la convierte en un marco analítico útil para entender los fenómenos que actúan y que caracterizan a la dinámica económica regional, nacional e internacional.

La idea fundamental a la que se refiere el ciclo económico es la existencia de un tipo de fluctuación en las actividades comerciales e industriales de las

1 En México se ha presentado un caso que no considera la existencia de una política regional: el Tratado de Libre Comercio de América del Norte (TLCAN) cuya firma no hace explícitos los impactos que éste tendría en las distintas regiones de México (Erquizio y Mendoza, 2008). 
economías que ocurren en periodos de ascensos y descensos simultáneos o de forma generalizada, con manifestación en distintas etapas ${ }^{2}$ y un carácter recurrente aunque no periódico. Como parte de este tipo de fluctuación de la actividad económica, las crisis se alejan de la idea comúnmente aceptada en la corriente ortodoxa que establece que los mecanismos de mercado son capaces y eficientes para conseguir los equilibrios de la economía, en donde el sistema económico puede autorregularse y evitar la existencia de crisis $-\mathrm{y}$, por ende, de ciclos económicos. ${ }^{3}$

En el caso de México, esfuerzos destacados sobre el estudio de los ciclos económicos son los trabajos realizados por Acevedo (2009), Loria (2008), Erquizio (2008), Cuadra (2008), Mejía (2003) y Torres (2000), entre otros. Si el estudio de los ciclos económicos es escaso, para el caso de los ciclos económicos regionales la escasez es aún más acentuada. Por ello, el interés particular del presente trabajo es conocer la posible existencia de ciclos regionales que permitan reflejar la dinámica económica en un espacio geográfico subnacional, en específico, la frontera norte de México.

En general, los espacios geográficos subnacionales han recibido mayor atención en otros aspectos del análisis económico; por ejemplo, se encuentran los análisis de crecimiento económico, convergencia-divergencia, distribución del ingreso, comercio internacional, organización industrial, mercados de trabajo, etcétera. ${ }^{4}$ Entre los economistas regionales se sabe que de un espacio geográfico a otro existen particularidades propias de cada uno de los territorios en cuanto a recursos -fuerza de trabajo, capital, tecnología, infraestructura, etcétera- y en cuanto a estructuras productivas -solvencia de los mercados, capacidad de crecimiento, etcétera-, que impactan y hacen diferente la dinámica subnacional (Zuccardi, 2002:3).

En la literatura económica se han distinguido dos formas de estudiar los ciclos regionales: la primera alude a la identificación de ciclos particulares,

2 Generalmente se habla de crisis, depresiones, recuperaciones y auges aunque no hay un consenso al respecto.

3 Esto se deriva del ensayo de Schumpeter (1927) titulado "Explicación de los ciclos económicos", en el cual retoma las ideas del trabajo de Arthur Pigou, Industrial Fluctuations.

4 También existen análisis de coyuntura más agregados sobre las variables macroeconómicas, marco institucional, competitividad, diagnósticos de una situación económica en un momento del tiempo, etcétera. 
analizando las fluctuaciones alrededor de la tendencia que muestran los ciclos de crecimiento y la capacidad potencial de crecimiento; la segunda se enfoca en los ascensos y descensos del indicador cíclico en términos absolutos, los así llamados ciclos clásicos que buscan capturar el grado de comovimiento del ciclo nacional y regional (Sherwood-Call, 1988:16).

El estudio de los ciclos como parte de la dinámica económica se caracteriza por considerar muchos aspectos de la economía que evolucionan día con día, pero su utilización es escasa en los estudios regionales. A pesar de su importancia y de su posible contribución al análisis regional, la principal limitante se encuentra en la existencia de información. ${ }^{5}$ Dado que no hay un indicador de alta frecuencia para la producción agregada por entidad federativa, en este estudio se utiliza como proxy del ciclo un indicador de empleo basado en las cifras de trabajadores permanentes y eventuales asegurados por el Instituto Mexicano del Seguro Social (IMSs).

No es difícil suponer que el empleo se mueve junto con la producción. Estudios empíricos señalan que los indicadores de empleo son procíclicos y siguen a la actividad productiva (Mejía, 2002:21). A partir de estas consideraciones se justifica el estudio de los ciclos económicos de las entidades federativas de la frontera norte de México con base en el análisis de las fluctuaciones del nivel de empleo. ${ }^{6}$

Quizá la dificultad más importante para describir los ciclos económicos de los estados de la frontera norte sea la supuesta sincronización de estas economías con el ciclo económico de Estados Unidos o con la economía de los estados del sur norteamericano, documentada en diversos estudios desde la década de los ochenta. Este comovimiento entre ambas economías está determinado a partir de la mayor concentración de exportaciones manufactureras que tienen como destino Estados Unidos y su concentración regional en los estados del norte de México (Félix y Dávila, 2008). La sincronización entre las economías de México y Estados Unidos parecería incuestionable si observamos los agregados macroeconómicos de ambos países en la era del TLCAN, sin embargo, si ésta fuera mayor en el caso de los estados de

5 El análisis dinámico requiere de información de series de tiempo largas y de alta frecuencia, la cual escasamente existe en las entidades federativas de México. No ocurre así para una escala nacional ya que se cuenta con más información de alta frecuencia.

${ }^{6}$ La disponibilidad de información ha definido el periodo de análisis. 
la frontera norte, cabe la posibilidad de que haya falta de sincronización o comovimiento entre ésta y la economía mexicana.

En ese sentido, la hipótesis de trabajo de la que partimos es que aquellas entidades federativas que tienen mayor participación en la contribución al crecimiento de la economía mexicana están más vinculadas con las fluctuaciones de la economía nacional, mientras que las que tienen una menor participación tienen una débil relación. Asimismo, dada la ubicación geográfica y los mayores flujos de comercio y de inversión por el proceso de integración económica con Norteamérica, los estados de la frontera norte muestran ciclos económicos vinculados con fenómenos asociados a choques internacionales.

El estudio se compone de tres apartados además de la introducción y las conclusiones. En el primero se revisan las diferentes teorías de los ciclos económicos y su importancia en la ciencia económica; en el segundo se revisa el tratamiento y la aplicación reciente de esta herramienta en el estudio de los ciclos económicos en México; y por último, en el tercer apartado se estiman los ciclos económicos en las economías de los estados de la frontera norte. Este ejercicio permite evaluar las características principales de las estructuras económicas de estos estados a partir del comportamiento que han observado en la última década. Con base en ello estamos en condiciones de predecir el comportamiento futuro de estas economías y las capacidades diferenciales para superar la crisis económica y retornar a una senda de crecimiento.

Las conclusiones apuntan a que no todos los estados podrán regresar a la tendencia de crecimiento que habían presentado antes de la crisis de fines de 2008. Las entidades en mejor posición para retomar el crecimiento son Nuevo León, Sonora y Tamaulipas. Por otro lado, también sugiere que la dinámica de corto plazo de los estados de la frontera norte tiene un distinto grado de comovimiento. Mientras algunas entidades muestran una estrecha relación con la economía nacional, en otras esta relación no es tan fuerte. Esto cobra sentido si consideramos que las actividades agregadas de los espacios subnacionales en el norte de México pueden estar ligadas a una dinámica regional distinta, puesto que existen cualidades geográficas que pueden insertarla en una dinámica diferente de la nacional; mientras otras, por su tamaño, su importancia y la capacidad de sus estructuras productivas, pueden ser más resistentes a choques externos a la región. Si bien las eviden- 
cias empíricas que de aquí se desprenden no son suficientes para establecer la existencia de ciclos económicos propios de las regiones, sí revelan que la manifestación regional de los ciclos económicos es diferente y se expresa en los distintos grados de comovimientos. Esto último exige seguir documentando los hechos característicos de los ciclos económicos a escala subnacional que puedan establecer su existencia.

En este sentido, este trabajo coincide en parte con la conclusión de Delajara (2010), quien encuentra un comovimiento cíclico alto en el empleo asociado a las fluctuaciones del empleo nacional, aunque esa estimación es para regiones y no para entidades federativas.

\section{Aspectos teóricos}

El fenómeno del ciclo económico parece tener una presencia indiscutible en cualquier economía capitalista (Sherman, 1991:163). El concepto de ciclo económico como un tipo de fluctuación de las economías implica aceptar la existencia de la inestabilidad del sistema económico, y ésa es la razón por la cual los modelos de crecimiento basados en la ortodoxia del equilibrio económico han dejado de lado ese tema. Las teorías que se encargan de explicar los ciclos son múltiples: van desde aquellas que se orientan según sus causas -sean éstas endógenas o exógenas-, las que las atribuyen a shocks del lado de la oferta o la demanda y las que consideran el equilibrio y el desequilibrio, hasta las que parten de la teoría económica o la economía política.

El modelo de multiplicador acelerador es una versión de los modelos macroeconómicos keynesianos popularizados después de la publicación de la Teoría General de Keynes. Esta tradición se deriva de la fundación de la sociedad econométrica que trata de cuantificar los hechos económicos. Por otra parte, los indicadores del ciclo económico del National Bureau of Economic Research (NBER) fueron impulsados por Mitchell y Burns para encontrar ciertas regularidades empíricas en diversas series económicas agregadas. Estas regularidades guardaron correspondencia con los modelos estructurales del ciclo y permitieron crear algoritmos de modelación económica. Por su parte, la teoría de los ciclos económicos reales representa un enfoque moderno para el estudio de este fenómeno. Las fallas de predicción de los modelos estructurales permitió la aparición de este enfoque bajo 
una concepción del equilibrio dinámico que ha dado impulso al debate y resurgimiento de la teoría de los ciclos económicos.

La teoría de Keynes (1936) responde a muchos de los desafíos de la teoría de los ciclos económicos. ${ }^{7}$ Buscando una explicación cuantitativa de los fenómenos que conciernen al ciclo económico (Kydland y Prescott, 1990:2), economistas como Frisch, Schumpeter y Fisher, entre otros, fundan la sociedad econométrica para fomentar la teoría económica cuantitativa, que tiene entre sus principales campos de estudio la teoría de los ciclos económicos. En paralelo a los modelos estructurales se encuentra la tradición de indicadores de la NBER, que en su definición de ciclo económico proponen que éstos son fluctuaciones en la actividad agregada y consisten en expansiones seguidas de recesiones generales, contracciones y recuperaciones que se unen a la fase de expansión del ciclo siguiente. "Esta secuencia de cambios es recurrente pero no periódica; la duración de los ciclos económicos varía desde más de un año hasta diez o doce años" (Burns y Mitchell, 1946:3).

\section{Ciclos económicos reales}

Los modelos del ciclo económico real constituyen una variación de los niveles agregados de producción por causas reales y representan una variación temporal del equilibrio competitivo. Las variaciones temporales del óptimo de Pareto están en función del comportamiento dinámico que muestra la tecnología y el stock de capital. A partir de una función de producción convencional del tipo Cobb-Douglas, donde $Y$ es el producto, $K$ el stock de capital, $L$ el trabajo y $A$ la tecnología, se expresa su transformación logarítmica como:

$$
\operatorname{In} Y_{t}=\alpha \operatorname{In} K_{t}+(1-\alpha)\left(\operatorname{In} A_{t}+\operatorname{In} L_{t}\right)
$$

Si se sabe que $K_{t}=\hat{s} Y_{t-1}$ y que $L_{t}=\hat{l} N_{t}$, entonces:

$$
\operatorname{In} Y_{t}=\alpha \operatorname{In} \hat{s} Y_{t-1}+(1-\alpha)\left(\operatorname{In} A_{t}+\operatorname{In} \hat{l}+\operatorname{In} L_{t}\right)
$$

\footnotetext{
7 De acuerdo con Keynes (1936:279): "El carácter esencial del ciclo y la regularidad de la secuencia en el tiempo, se debe a las fluctuaciones de la eficiencia marginal del capital [...] y se profundiza por otras variables importantes en un periodo breve del sistema económico".
} 
Siendo $N$ el tamaño de la población y $g$ la tasa de crecimiento del capital.

$\operatorname{Sin} A_{t}=\bar{A}+g_{t}+\tilde{A}_{t} \quad \mathrm{y}$

$\operatorname{In} N_{t}=\bar{N}+n_{t}$, entonces:

$\operatorname{In} Y_{t}=\alpha \operatorname{In} \hat{s}+\alpha \operatorname{In} Y_{t-1}+(1-\alpha)\left(\bar{A}+g_{t}\right)(1-\alpha) \tilde{A}_{t}+\operatorname{In} \hat{l}+\bar{N}+n$

En (3) debe resaltarse que hay dos elementos que no siguen una trayectoria determinista: $\alpha \operatorname{In} Y_{t-1}$ y $(1-\alpha) \tilde{A}_{t}$. En consecuencia, puede señalarse lo siguiente:

$$
\tilde{Y}_{t}=\alpha \tilde{Y}_{t-1}(1-\alpha) \tilde{A}_{t}
$$

Ahora, si se sustituye en $\tilde{A}_{t}$ según (2), se obtiene:

$$
\tilde{Y}_{t}=\alpha \tilde{Y}_{t-1}(1-\alpha)\left(\rho_{A} \tilde{A}_{t-1}+\varepsilon_{A, T}\right)
$$

Dado que $\tilde{A}_{t-1}=\frac{\tilde{Y}_{t-1}+\alpha \tilde{Y}_{t-2}}{1-\alpha}$ se puede obtener:

$$
\tilde{Y}_{t}=\left(\alpha+\rho_{A}\right) \tilde{Y}_{t-1}-\alpha \rho_{A} \tilde{Y}_{(t-2)}+(1-\alpha) \varepsilon_{A, T}
$$

La formulación de (6) representa las perturbaciones que origina la desviación de la producción en torno a su senda tendencial de crecimiento. ${ }^{8}$ Este planteamiento es una discusión iniciada por el trabajo de Nelson y Plosser (1982) y es la representación empírica que han adoptado los modelos del ciclo económico real.

En particular, se ha señalado que la estimación de regresiones de series de tiempo desprovistas de tendencia lleva a interpretar los residuales como el componente cíclico, sin embargo, el movimiento secular no necesita ser modelado por una tendencia determinista. De acuerdo a Nelson y Plosser (1982:140), si el movimiento en las series de tiempo macroeconómi-

8 Es un proceso autorregresivo de segundo orden con ruido blanco. 
cas es estocástico en lugar de determinista, entonces los modelos basados en tendencias residuales están mal especificados. ${ }^{9}$ La evidencia empírica encontrada muestra que la mayoría de series de tiempo macroeconómicas contienen un componente secular que sigue un proceso estocástico en lugar de determinista. Más aún, la desviación estándar de las innovaciones en el componente secular es más grande que la desviación estándar de las innovaciones en el componente cíclico. ${ }^{10}$ Es decir, las variaciones en el producto son dominadas por los cambios en el componente secular más que en el componente cíclico (Nelson y Plosser, 1982:155). Este hallazgo empírico tiene algunas implicaciones para la teoría de los ciclos económicos. Generalmente, las fluctuaciones del producto son consecuencia de distorsiones monetarias que sólo tienen un impacto transitorio sin efectos reales permanentes. Sin embargo, la varianza mayor del componente secular en relación con el componente cíclico sugiere que los disturbios reales (no monetarios) son probablemente una fuente más importante de fluctuaciones en el producto que los disturbios monetarios (Nelson y Plosser, 1982:155).

En este sentido, las primeras preocupaciones de la agenda de investigación de los ciclos económicos reales trataba de buscar las causas que determinan las fluctuaciones alrededor de la tendencia estocástica del producto, figurando las perturbaciones de la tecnología y la sustitución intertemporal de la oferta de trabajo como elementos explicativos importantes (Kydland y Prescott, 1982:1366).

Fundamentalmente, los esfuerzos de esta agenda encontraron que las variaciones del residuo de Solow ${ }^{11}$-es decir, los disturbios tecnológicosdan cuenta de más de la mitad de las fluctuaciones del producto de la economía de Estados Unidos. Si los shocks de productividad son permanentes, entonces cada uno determina una nueva trayectoria de crecimiento; por

\footnotetext{
9 El tipo de incorrecta especificación que surge de una inapropiada serie sin tendencia puede ilustrarse considerando las propiedades de los residuos de una regresión con caminata aleatoria en el tiempo.

10 Las innovaciones son parte del componente permanente que sigue una caminata aleatoria.

11 El residuo de Solow mide la tasa de progreso técnico considerando el porcentaje de variación del producto menos el porcentaje de variación de los insumos. Los insumos se ponderan de acuerdo con la participación del factor en la función de producción.
} 
ello se dice que es un modelo real. Estos resultados asumen un supuesto en el que los trabajadores laboran el mismo número de horas y el número de trabajadores no varía12 (Kydland y Prescott, 1982:1367).

La novedad en esta teoría es que los ciclos pueden explicarse utilizando un enfoque del equilibrio competitivo que normalmente se manifiesta como la fluctuación del producto en torno a su senda tendencial. La concepción del equilibrio se establece a través de la sustitución intertemporal de la oferta de trabajo, determinada por los niveles de salario relativo de periodo a periodo y la tasa de interés real. Sin embargo, la conducta de optimización que genera fluctuaciones de la oferta de trabajo es una manifestación, más no una causa; la causa que produce la desviación de la producción en torno a su senda tendencial son las perturbaciones tecnológicas, las cuales a su vez modifican el pago a los factores de producción y las decisiones racionales de los agentes económicos, produciendo así fluctuaciones en la actividad económica. Es decir, son de índole real puesto que los disturbios monetarios no afectan a la producción, ya que solo tienen efectos transitorios. En específico, se ha encontrado que la mayoría de las fluctuaciones en el producto se deben a los cambios en el residuo de Solow, que es la medida de las perturbaciones tecnológicas.

Esta concepción sobre el origen de las fluctuaciones no ha estado exenta de controversias. Primero, si el residuo de Solow es altamente cíclico, entonces disminuye cuando cae la producción, por ende, las fases recesivas implican un proceso de regresión tecnológica. Segundo, esta regresión tecnológica hace que baje el producto marginal del trabajo, por tanto bajan los salarios; luego, si bajan los salarios, los individuos deciden reducir su tiempo de trabajo e incrementar el ocio. Sin embargo, algunas evidencias empíricas muestran poca disposición de los individuos a sustituir ocio en el tiempo; por tanto, el mecanismo fundamental a través del cual se generan las fluctuaciones es poco convincente para sostener el planteamiento que postula la teoría de los ciclos económicos reales. Tercero, esta teoría parece olvidar los mecanismos de intercambio debido a que las recesiones se caracterizan precisamente por la interrupción de este proceso. Las empresas no pueden vender sus productos y los trabajadores quieren obtener esas mercancías a cambio de su trabajo, sin embargo, el intercambio no tiene lugar.

12 Alude al supuesto de agentes económicos (hogares) homogéneos. 
Los planteamientos que se desprenden de la teoría de los ciclos económicos reales tienen implicaciones controvertidas, no obstante, ha logrado despertar el debate entre las corrientes de pensamiento económico. Esto, sin duda, ha permitido enriquecer la teoría de los ciclos y convertirla en un marco analítico valioso para evaluar el desempeño que muestran las distintas economías. La definición y adopción de las ideas que de ella se desprenden, así como las críticas correspondientes, son cuestiones que deben considerarse al momento de interpretar los fenómenos económicos.

\section{El ciclo económico en la frontera}

Los estudios del ciclo económico en México se han concentrado en verificar la existencia de ciclos económicos e identificarlos, así como encontrar algunas regularidades empíricas del fenómeno o hechos estilizados. En la historia reciente del país se han presentado distintas crisis económicas que los estudiosos de la economía mexicana han logrado identificar. Algunos estudios que retoman la metodología popularizada por Kydland y Prescott para analizar los ciclos económicos son los desarrollados por Mejía (2002, 2003a, 2003b). Otros trabajos que analizan la morfología del ciclo económico son los de Erquizio (2008, 2007a y b, 2006a y b, 2003, 2001), quien identifica las fases de los ciclos clásicos y de crecimiento, así como las características de los mismos en términos de duración, volatilidad, regularidad y amplitud. Loria y colaboradores (2008) se proponen hacer un cálculo más riguroso de la capacidad real de crecimiento de la economía mexicana. A través de modelos estructurales de series de tiempo, estos autores estiman el producto potencial, la brecha del producto y los ciclos económicos en México durante el lapso 1980-2006 (Loria et al., 2008:29). Por su parte, Acevedo (2009) estima la capacidad de crecimiento de la economía mexicana y compara los resultados obtenidos con el filtro HP utilizando una función de producción Cobb-Douglas y el filtro de Kalman para determinar el nivel de empleo que corresponde con la tasa natural de desempleo.

La importancia económica de la región fronteriza norte ha crecido conforme avanzó en el país el proceso de descentralización de la actividad económica desde la década de los ochenta, y más recientemente, a partir de la mayor profundización alcanzada con el proceso de integración económica posterior a la firma del Tratado de Libre Comercio de América del Norte (TLCAN) hacia mediados de los noventa. 
El cuadro 1 muestra que la contribución económica regional de la frontera norte en la producción nacional es casi una cuarta parte con $23.5 \%$ de participación. La entidad federativa más importante es, sin duda, Nuevo León, estado que aporta $6.9 \%$, seguido por Chihuahua con $4.3 \%$, Coahuila con $3.28 \%$, Baja California con 3.2\%, Tamaulipas con $3.04 \%$ y Sonora con 2.78 por ciento.

Cuadro 1

Indicadores de producción 1993-2006

\begin{tabular}{|c|c|c|c|c|c|c|c|c|}
\hline 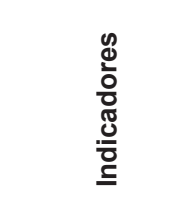 & 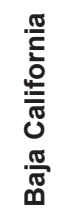 & 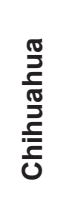 & $\begin{array}{l}\frac{\pi}{\pi} \\
\frac{2}{\pi} \\
0 \\
ن\end{array}$ & 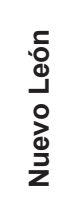 & 임 & 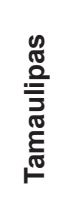 & 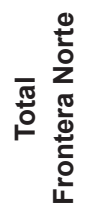 & $\begin{array}{l}\bar{\pi} \\
\frac{0}{0} \\
\frac{0}{0} \\
\frac{\pi}{2}\end{array}$ \\
\hline $\begin{array}{l}\text { PIB promedio } \\
\text { millones } \\
\text { de pesos } 2003\end{array}$ & 44.3 & 59.9 & 45.7 & 96.2 & 38.7 & 42.3 & 327.2 & 1392.4 \\
\hline $\begin{array}{l}\text { Participación } \\
(\%)\end{array}$ & 3.2 & 4.3 & 3.3 & 6.9 & 2.8 & 3.0 & 23.5 & 100.0 \\
\hline $\begin{array}{l}\text { Crecimiento } \\
\text { promedio (\%) }\end{array}$ & 4.6 & 4.3 & 4.3 & 4.2 & 3.9 & 4.0 & 4.2 & 3.0 \\
\hline
\end{tabular}

Fuente: INEGI.

Por otra parte, el crecimiento económico de las entidades federativas del norte de México se ubica por encima del mostrado por el desempeño nacional. La frontera norte en su conjunto crece a ritmo de $4.2 \%$ anual, mientras el país lo hace a $2.9 \%$ en el mismo periodo. Baja California es la entidad con mayor dinamismo pues crece a $4.6 \%$, seguida de Coahuila, Chihuahua y Nuevo León con $4.3 \%, 4.2 \%$ y $4.1 \%$ respectivamente. Por último, los estados más rezagados son Tamaulipas con $3.9 \%$ y Sonora con 3.8 por ciento.

Esta caracterización de la frontera norte es particularmente importante, en la medida que revela el tamaño y la importancia de cada Entidad Federativa así como de la región en su conjunto. En este sentido, se contextualiza su desempeño en relación a la actividad económica nacional. 


\section{Cuadro 2}

Indicadores de la producción manufacturera 1993-2006

\begin{tabular}{|c|c|c|c|c|c|c|c|c|}
\hline 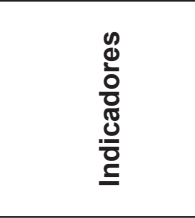 & 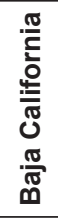 & 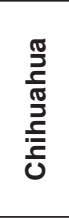 & $\begin{array}{l}\frac{\pi}{5} \\
\frac{\pi}{\pi} \\
0 \\
0\end{array}$ & 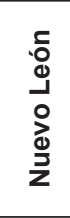 & 흠 & 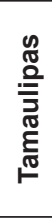 & 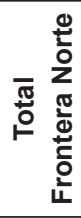 & $\begin{array}{l}\bar{\pi} \\
\frac{0}{0} \\
\frac{\pi}{2} \\
z\end{array}$ \\
\hline $\begin{array}{l}\text { PIB promedio } \\
\text { millones } \\
\text { de pesos } 2003\end{array}$ & 8.9 & 12.0 & 16.6 & 25.9 & 6.7 & 8.9 & 79.0 & 281.0 \\
\hline $\begin{array}{l}\text { Participación } \\
(\%)\end{array}$ & 3.2 & 4.3 & 5.9 & 9.2 & 2.4 & 3.2 & 28.1 & 100.0 \\
\hline $\begin{array}{l}\text { Crecimiento } \\
\text { promedio (\%) }\end{array}$ & 5.1 & 3.8 & 5.8 & 4.2 & 4.4 & 5.2 & 4.7 & 3.2 \\
\hline
\end{tabular}

Fuente: INEGI.

Las vocaciones productivas de la economía de la frontera norte son de carácter industrial. Las particularidades de la región, las características geográficas, recursos naturales y la zona desértica, son sólo algunas de las cualidades que impiden el desarrollo de otras actividades productivas.

En cambio, la vecindad con Estados Unidos, las vías de comunicación, la infraestructura física y de capital humano son factores que han favorecido el desarrollo industrial de la región. Para observar la importancia del sector industrial de la economía de la frontera norte en la actividad industrial nacional, se observa la participación de la industria manufacturera de la región en relación con el sector manufacturero del país.

En el cuadro 2 se aprecia que la industria manufacturera en el norte de México contribuye con $28.1 \%$ del total nacional. Del mismo modo, Nuevo León cuenta con la economía industrial más fuerte aportando 9.2\%, seguido de Coahuila con 5.9\%, Chihuahua con $4.27 \%$, Baja california con $3.17 \%$, Tamaulipas con $3.16 \%$ y Sonora con 2.38 por ciento. 
Cuadro 3. Personal ocupado promedio 1994-2008 Estados de la frontera norte y nacional, total e industria manufacturera

\begin{tabular}{|l|c|c|c|c|}
\hline & \multicolumn{2}{|c|}{ Empleo total } & \multicolumn{2}{c|}{ Empleo manufacturero } \\
\hline Baja California & 596.6 & 4.7 & 17402.9 & 1.3 \\
\hline Chihuahua & 648.2 & 5.1 & 57262.3 & 4.3 \\
\hline Coahuila & 496.2 & 3.9 & 19090.3 & 1.4 \\
\hline Nuevo León & 976.9 & 7.7 & 160601.7 & 12.0 \\
\hline Sonora & 372.1 & 2.9 & 20916.9 & 1.6 \\
\hline Tamaulipas & 515.2 & 4.1 & - & - \\
\hline $\begin{array}{l}\text { Total frontera } \\
\text { norte }\end{array}$ & 3605.2 & 28.4 & 257871.2 & 19.3 \\
\hline Nacional & 12703.5 & 100.0 & 1336936.0 & 100.0 \\
\hline
\end{tabular}

Fuente: Elaboración propia con datos de la Encuesta Industrial Mensual, INEGI e Instituto Mexicano del Seguro Social.

Los datos presentados revelan el fuerte carácter industrial de la frontera norte y lo importante que ésta es para la industria nacional. Más aún, el desempeño mostrado por la región en su conjunto y cada una de sus entidades indica un crecimiento mucho mayor al presentado por la industria manufacturera nacional.

Para observar qué impactos tiene la industria en la generación de empleos para la población de la frontera norte se presenta el número de personas ocupadas en la industria manufacturera y la participación porcentual de las mismas en el total nacional. Debido a que la encuesta industrial no ofrece el dato para Tamaulipas, se presentan sólo los cinco estados restantes. El cuadro 3 revela que cinco de los seis estados de la frontera norte producen alrededor de $20 \%$ del empleo que genera la manufactura nacional. La entidad con mayor aporte a la generación de empleo es Nuevo León con 12\%, seguida por Coahuila con $4.28 \%$, Sonora con $1.56 \%$, Chihuahua con $1.42 \%$ y Baja California con $1.3 \%$. Si se incluye Tamaulipas, esta cantidad podría ubicarse en alrededor de una cuarta parte del empleo generado por el país. Los resultados anteriores pueden encontrar correspondencia si se observa el comportamiento del empleo total de cada entidad en relación con el total nacional.

Efectivamente, en el cuadro 4 se aprecia que la región frontera norte en su conjunto crea más de una cuarta parte del empleo total generado por el país, puesto que contribuye con $28.3 \%$. Nuevo León es el estado más 
importante en este rubro con $7.6 \%$, seguido por Chihuahua con $5.1 \%$, Baja California con $4.69 \%$, Tamaulipas con $4.05 \%$, y Coahuila y Sonora con 3.9 y $2.92 \%$ respectivamente.

\section{Metodología}

El análisis de los ciclos económicos en las entidades de la frontera norte siguió tres procesos: primero, se evaluó la viabilidad de utilizar una variable que aproxime el comportamiento cíclico del producto mediante análisis de correlación; después, se realizó el análisis para la descomposición de la tendencia y ciclo de la serie de tiempo con el filtro HP, de amplia utilización en la literatura especializada; y posteriormente se emprendió el análisis de las series de tiempo estatales en relación con las series nacionales con un procedimiento que explicamos a continuación.

El objetivo se dirige a observar los comovimientos entre la economía nacional y regional. Varios trabajos referidos a los estudios regionales del ciclo económico han explorado las diferencias entre los picos y los valles del empleo de varios estados. Esto es particularmente útil en la medida en que permite analizar ciclos específicos, sin embargo, no proporciona una visión conjunta del fenómeno. El objetivo se consigue a través de la construcción de un modelo de vectores autorregresivos VAR. El enfoque de los modelos VAR es un sistema libre sin una estructura teórica subyacente. Siguiendo a Zuccardi (2002), es posible establecer una medida de fuerza de cohesión denominada LiNK por Sherwood-Call (1988) con base en la descomposición de varianza del sistema VAR que busca reflejar la semejanza de la dinámica entre el país y sus regiones. En particular, se construye un sistema de ecuaciones con las siguientes características:

$$
\begin{aligned}
& \Delta E_{\text {Mex }- \text { Reg }, t}=\alpha_{1}+\sum_{i=1}^{p} \beta_{i 1} \Delta E_{\text {Mex-Reg, }, t-i}+\sum_{i=1}^{q} \eta_{i 1} \Delta E_{\text {Reg }, t-i}+e_{\text {Mex }-\operatorname{Reg}, t} \\
& \Delta E_{\text {Reg, } t}=\alpha_{2}+\beta_{02} \Delta E_{M e x-\operatorname{Reg}, t}+\sum_{i=1}^{p} \beta_{i 2} \Delta E_{M e x-\operatorname{Reg}, t-i}+\sum_{i=1}^{q} \eta_{i 2} \Delta E_{\text {Reg }, t-i}+e_{\text {reg }, t}
\end{aligned}
$$

Donde $\Delta E_{\text {Mex }-\operatorname{Reg}, t}$ representa la variación del empleo nacional menos el empleo de la región; $\Delta E_{R e g, t}$ es la variación del empleo de la región; $e_{M e x-R e g, t}$ es 
un error estocástico que afecta a todo el país contemporáneamente; y $e_{\text {Reg, },}$ es un error estocástico que afecta sólo a la región. Debe notarse que en la ecuación (8) el empleo nacional afecta de forma contemporánea a la región, lo cual incluye los choques estocásticos que afectan al país y por ende a sus regiones, o en otras palabras, una perturbación a nivel nacional puede afectar a una región específica, empero, es difícil que los choques que afectan a esta región afecten a la nación en su conjunto de forma contemporánea13 (Zuccardi, 2002:19). Por tanto, si se sustituye (7) en (8) se tiene:

$$
\begin{aligned}
\Delta E_{\text {Reg, }, t}= & \left(\alpha_{2}+\beta_{02} \alpha_{1}\right)+\sum_{i=1}^{p}\left(\beta_{i 2+} \beta_{02} \beta_{i 1}\right) \Delta E_{\text {Mex }-R e g, t-i} \\
& +\sum_{i=1}^{q}\left(\eta_{i 2}+\beta_{02} \eta_{i 1}\right) \Delta E_{\text {Reg, },-i} \ldots+\beta_{02} e_{M e x-R e g}+e_{R e g, t}
\end{aligned}
$$

De este modo, la ecuación (9) está explicada por los retardos del empleo nacional y regional y por los choques que afectan a la región y a la nación en su conjunto. Por tanto, el sistema de ecuaciones establecido por (7) y (8) queda exactamente identificado ${ }^{14}$ y puede reformularse de la siguiente manera:

$$
\begin{aligned}
& \Delta E_{\text {Mex-Reg, } t}=\alpha_{1}+\sum_{i=1}^{p} \beta_{i 1} \Delta E_{\text {Mex }-\operatorname{Reg}, t-i}+\sum_{i=1}^{q} \eta_{i 1} \Delta E_{\text {Reg, } t-i}+e_{\text {Mex }-\operatorname{Reg}, t} \\
& \Delta E_{\text {Reg, } t}=\left(\alpha_{2}+\beta_{02} \alpha_{1}\right)+\sum_{i=1}^{p}\left(\beta_{i 2}+\beta_{02} \beta_{i 1}\right) \Delta E_{\text {Mex }- \text { Reg, }, t-i} \\
& +\sum_{i=1}^{q}\left(\eta_{i 2}+\beta_{02} \eta_{i 1}\right) \Delta E_{R e g, t-i} \ldots+\beta_{02} e_{M e x-\operatorname{Reg}}+e_{R e g, t}
\end{aligned}
$$

13 Aunque quizá lo haga de forma retardada.

14 Recuérdese que en un sistema VAR como el siguiente:

$$
Y_{t}=\alpha+\beta_{0} Y_{t}+\beta_{1} Y_{t-1}+\beta_{2} Y_{t-2}+\ldots \beta_{p} Y_{t-p}+\varepsilon_{t}
$$

la matriz es de suma importancia porque es una matriz de coeficientes que determina el efecto contemporáneo de un choque en una determinada variable sobre las demás en el sistema. Si esta matriz es diagonal, un choque sólo tiene efectos contemporáneos sobre esa variable, si no lo es, tiene efectos sobre las demás variables generando un sistema inestable. En este caso, el sentido establecido de los choques nación-región, región-nación ha permitido la formulación 2.5 que alivia este inconveniente. 
De acuerdo con este sistema de ecuaciones, la variación del empleo regional y nacional se explica por el empleo pasado nacional, la variación del empleo regional en $t$ periodos atrás y el error estocástico que afecta a toda la nación y a la región. Entonces, a través del modelo var es posible establecer una relación entre el empleo nacional y regional capaz de revelar el grado de comovimiento de ambas variables.

Este procedimiento calcula la varianza del error de pronóstico para un periodo determinado hacia adelante, después de someter al sistema a un choque estocástico. En cada periodo se obtiene el porcentaje de la varianza del error de pronóstico de cada una de las variables involucradas en el sistema. Esa medida es la que se conoce como LINK y con ella es posible conocer los movimientos comunes entre la economía regional y nacional.

\section{Estimación y resultados}

Observar los ciclos económicos a nivel regional se convierte en una tarea muy complicada pues más allá de que éste sea un fenómeno inobservable, se enfrenta el problema de disponibilidad y consistencia de las series a utilizar. La información que publica el Instituto Nacional de Estadística, Geografía e Informática (INEGI) para la producción estatal tiene un retraso importante que impide observar las fluctuaciones de corto plazo. Además, los datos más recientes para el producto interno bruto (РIB) estatal durante los lapsos 2003-2008 y 1993-2006 son series generadas por metodologías distintas que arrojan algunas inconsistencias. Esto es debido a que si se pretende realizar un enganche entre las mismas, los resultados son evidentemente incongruentes. Incluso el dato de 2008 muestra que la tasa de crecimiento con respecto al año anterior es positiva, lo cual no indica ninguna influencia de la recesión que inicia en 2008 sobre las economías estatales.

La naturaleza de los datos en relación con su disponibilidad y consistencia ha ocasionado que esta investigación opte por elegir una variable proxy de la producción que permite observar la dinámica de corto plazo y, sobre todo, es consistente con algunos sucesos de la historia reciente de la economía mexicana y de las economías estatales de la frontera norte. La variable que se ha elegido como proxy de la producción y que puede ser de utilidad para observar las oscilaciones de los ciclos económicos regionales es el 
empleo. ${ }^{15}$ En el apartado contextual se ha mostrado que un hecho estilizado para la economía mexicana muestra al empleo como una variable que sigue a la producción y es procíclica. Si hacemos válido este hecho podemos suponer que el empleo es capaz de dibujar los ciclos que muestran las economías. Una forma de corroborar este hecho y tener una aproximación a lo que se plantea se basa en la alta correlación que presentan las series del PIB y empleo para el periodo 1997.3-2010.1. Los resultados se presentan en el cuadro 4.

Cuadro 4. Análisis de correlación PIB-ocupación nacional y ocupación nacional-estatal en la frontera norte

\begin{tabular}{|l|c|c|c|c|c|}
\hline \multicolumn{1}{|c|}{ Entidad } & PIB & $\begin{array}{c}\text { Ocupación } \\
\text { nacional }\end{array}$ & $\begin{array}{c}\text { Ocupación } \\
\text { estatal }\end{array}$ & t-estadístico & Probabilidad \\
\hline Nacional & \multicolumn{2}{|c|}{0.8265} & 10.270 & 0.0000 \\
\hline Baja California & \multicolumn{2}{|c|}{0.894924} & 14.039 & 0.0000 \\
\hline Chihuahua & & 0.355518 & 2.663 & 0.0150 \\
\hline Coahuila & & 0.861209 & 11.861 & 0.0000 \\
\hline Nuevo León & & 0.996047 & 78.497 & 0.0000 \\
\hline Sonora & & 0.972826 & 29.411 & 0.0000 \\
\hline Tamaulipas & & 0.958787 & 23.621 & 0.0000 \\
\hline
\end{tabular}

Fuente: Elaborado con datos de INEGI e IMSs.

Como puede notarse, la relación refleja un alto coeficiente de correlación entre el PIB y el empleo, equivalente a $82 \%$. La relación es estadísticamente significativa con un estadístico $t$ de 10.27 y una probabilidad menor al $5 \%$. Ahora bien, es posible relacionar el empleo nacional con el regional. El cuadro 5 muestra una alta correlación entre el empleo nacional y empleo de las entidades de la frontera norte. Si se observan las razones $t$ y la probabilidad asociada, se encuentra que las relaciones son estadísticamente significativas al 95\% de confianza. Chihuahua es el estado que muestra una correlación más baja, pero su significancia estadística tiene el mismo nivel de confianza.

Los estudios empíricos toman el producto como indicador del ciclo. Si el PIB nacional muestra alta correlación con el empleo nacional y este último a

15 Ya existen estudios en otros países que han optado por esta vía. Los datos que aquí se toman se refieren al número de trabajadores permanentes y eventuales afiliados al Instituto Mexicano del Seguro Social. 
su vez muestra alta correlación con el empleo de las entidades federativas de la frontera norte, es posible establecer que el empleo es una variable indicativa de los ciclos a nivel regional. A continuación presentamos la tendencia de crecimiento de los estados del norte de México utilizando el filtro HP.

Como se observa en el diagrama 1, durante el lapso 1997.7-2010.4 existen dos ciclos económicos en el estado de Baja California: ${ }^{16}$ el primero se presenta en 1997.7-2003.8 y el segundo en 2003.9-2009.6. El ascenso del primer ciclo duró 42 meses, mientras el segundo tuvo una duración de 51 meses. De la misma manera, se identificaron dos periodos de descenso: el primero se encuentra en 2001.1-2003.8 y el segundo en 2008.1-2009.6. El primer periodo de descenso tuvo una duración de 32 meses y el segundo de 15. Estas características permiten observar que los ciclos identificados para Baja California ocurrieron más o menos al mismo tiempo de los que encontraron a escala nacional.

La recesión de 2001-2003 impactó a los niveles de bienestar de la población de Baja California. Los impactos negativos de la recesión se tradujeron en la pérdida de 42311 empleos formales, siendo la industria de la transformación la que explica en mayor medida esta caída. Para la recesión de 20082009 la pérdida de bienestar medida en número de empleos se tradujo en una reducción de 67331 plazas de trabajo, superior a la mostrada por la recesión anterior. La industria de la transformación evidenció un mayor retroceso con 49101 despidos, seguida de la industria de la construcción con 11526.

Otra característica importante que se debe señalar es la profundidad y severidad a la que han ocurrido las recesiones. Acevedo (2009) señala que la primera se mide por el grado de desaceleración que sufren las variables reales, en tanto la segunda se refiere a la velocidad con que ocurre el descenso. Considerando ambas recesiones, se puede establecer que la de 20082009 generó una mayor profundidad y severidad debido a que produjo una mayor pérdida de empleos y ocurrió con más velocidad.

Uno de los aspectos más interesantes del diagrama es que la última recesión debilitó de manera importante la capacidad potencial de la economía para generar empleos, debido a que los niveles de empleo en 2008-2009

16 Dado que no se cuenta con información precedente a 1997.7, no se podrá determinar el valle inicial del primer ciclo económico de los estados de la frontera norte. Por tanto, los primeros ciclos estarán incompletos. 

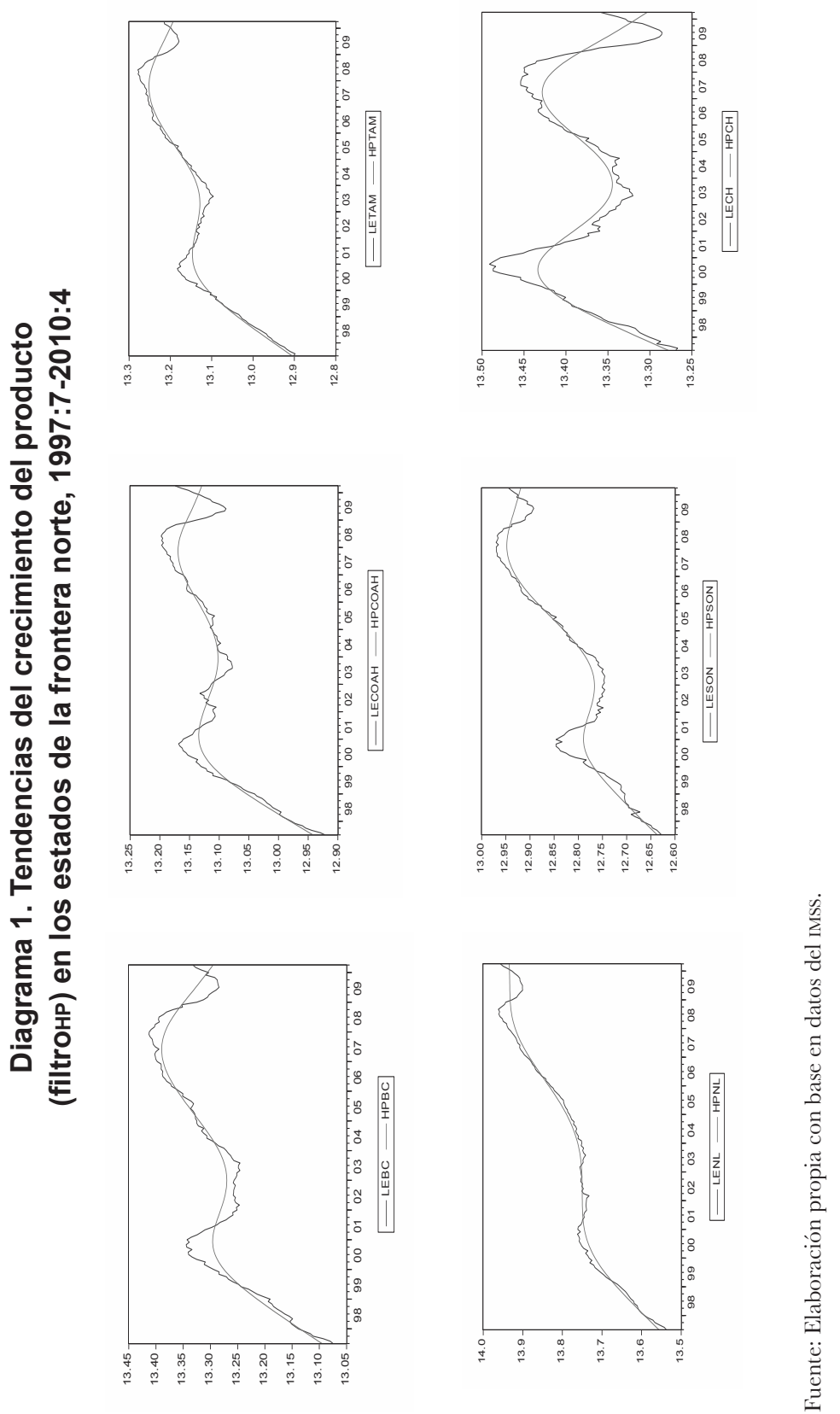

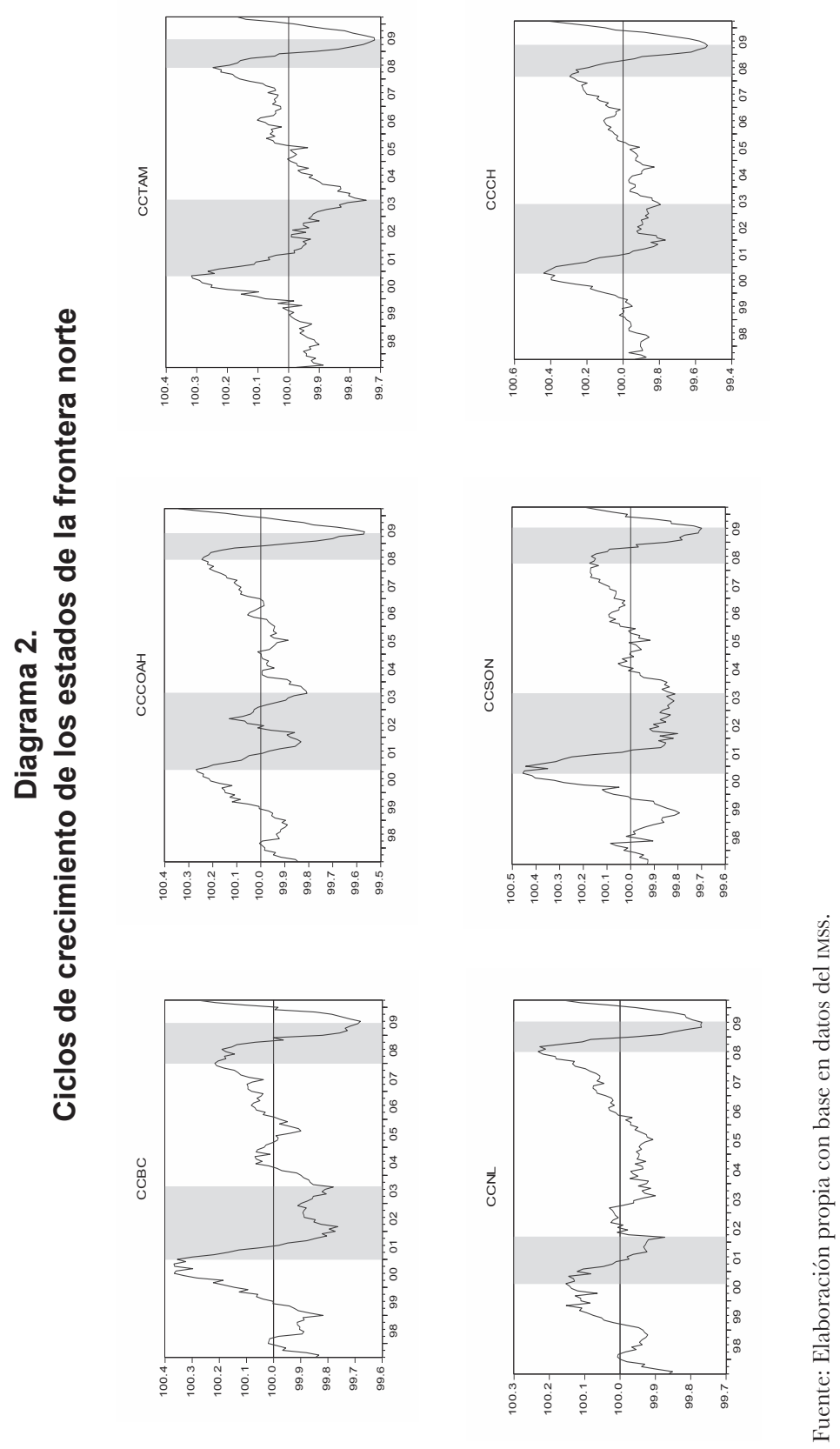
cayeron por debajo de los niveles alcanzados por el pico (2001.1) del ciclo anterior y ello revirtió la pendiente del componente de tendencia expresada por el filtro HP.

Esto es un reflejo de la subutilización de recursos en que está incurriendo la economía de esta entidad dados los choques endógenos y exógenos a los que enfrenta; por ello, es necesario aplicar políticas económicas regionales que subsanen la problemática que enfrenta a fin de volver a alcanzar los niveles óptimos en el uso de recursos que ha estado empleando.

El comportamiento cíclico referente a Coahuila, cuyo gráfico se localiza en la segunda posición superior del diagrama 1, muestra que durante el lapso 1997.7-2010.1 la economía de Coahuila presentó dos ciclos económicos: el primero se fecha en el periodo 1997.7-2003.8 con una duración de 74 meses y el segundo durante 2003.9-2009.5 durante 68 meses. La duración del primer ascenso es de 41 meses, mientras la del segundo es de 57 . Los periodos de descenso se presentaron en el lapso 2000.11-2003.8 para el primer ciclo y en 2008.6-2009.5 para el segundo. La duración de estos declives fue de 33 meses para el primer ciclo y 11 meses para el segundo.

La recesión de 2001-200317 representó una pérdida de 37344 puestos de trabajo para la economía del estado. Los sectores que produjeron esta situación fueron la industria de la transformación -que disminuyó en 33429 el nivel de empleos-, la industria de la construcción -que redujo 5317 empleos- y servicios para empresas, personas y el hogar -que perdió 4873 plazas-. Por su parte, la recesión de 2008-2009 se tradujo en la pérdida de 55073 empleos. La industria de la transformación produjo el despido de 37406 personas, la construcción 11607 y el comercio, que no había sido afectado en la anterior recesión, en esta ocasión produjo 3064 despidos. Servicios para empresas, personas y el hogar perdió 4873 empleos. Se puede establecer que la recesión 2008-2009 fue más profunda que la de 2001-2003 debido a que se perdió un mayor número de empleos. También se puede decir que ésta fue más severa debido a que ocurrió con una mayor velocidad.

Asimismo, se observa que el filtro HP tuvo un cambio de pendiente que manifiesta la capacidad que perdió la economía para generar empleos; de la misma forma, se aprecia que otra de las debilidades de la economía es la

17 Debe destacarse que durante esta recesión la economía logró revertir las pérdidas de empleo que estaba sufriendo, sin embargo, no salió avante y volvió a mostrar un declive. 
caída por debajo de la situación alcanzada por el pico (2000.11) del ciclo anterior. Esto último se debe sobre todo a que la economía no logró restablecer la dinámica de empleos generada en el primer ciclo, lo cual se observa en una pendiente más plana durante el ascenso del segundo ciclo. Sin embargo, durante el segundo trimestre del 2009 la economía parece entrar en recuperación en lo que concierne a la generación de empleos. No obstante, si sólo se observa el indicador del ciclo de crecimiento, la recuperación de la economía puede parecer engañosa ya que el indicador ha rebasado su senda tendencial de crecimiento que está con una pendiente descendente. Si bien esta economía ha logrado recuperarse en la generación de empleos, apenas ha logrado restablecer el nivel alcanzado por el pico del ciclo anterior (2000.11).

Por lo anterior, debe hacerse una lectura cuidadosa de la capacidad potencial para la generación de empleos establecida por el filtro HP. Esto es importante para efectos de política económica ya que las tendencias generales establecen que la economía aún mantiene una subutilización de los recursos que posee. Esta lectura puede corroborarse si se considera el desempeño mostrado por el personal ocupado en la industria manufacturera. Este hecho presentado en esta economía regional habla de las dificultades que se han enfrentado en cuanto a la generación de empleos. Parece entonces que la capacidad de la industria manufacturera para contribuir a la economía en su conjunto no ha conseguido restablecer su dinamismo para ser el motor de crecimiento.

Respecto a los ciclos de crecimiento de la economía de Nuevo León durante el lapso 1997.7-2010.4, se observa en la primera gráfica inferior del diagrama la existencia de dos ciclos económicos. El primero se encuentra en 1997.7-2002.3 y el segundo en 2002.4-2009.7. La duración de sus ascensos es de 38 meses para el primer ciclo y de 73 meses para el segundo. Las diferencias en la duración de ambos ascensos provienen de la falta de información precedente a 1997.7 para el primer ciclo.

En cuanto a las recesiones, se establece que la observada durante el primer ciclo se fecha en 2000.8-2002.3 con una duración de 18 meses, mientras la segunda se presenta durante el lapso 2008.6-2009.6 con una duración de 12 meses. Este hecho revela la capacidad que tiene esta economía para salir de las recesiones.

Ahora bien, si se observa la recesión de 2000-2002 se encontrará que ésta produjo el despido de 16886 trabajadores. Este suceso -que se ha convertido 
en un hecho recurrente en las economías estatales de la frontera norte- se derivó por la contracción observada en la industria de la transformación, sin embargo, logró contrarrestarse por el desempeño mostrado por otros sectores.

Para la recesión de 2008-2009, la economía incurrió en una pérdida de 63434 empleos, propiciados principalmente por 38004 de la industria de la transformación, 12603 de la industria de la construcción, 6330 de servicios para empresas, personas y el hogar y 5834 del comercio. Otro hallazgo que puede señalarse es el hecho de que la recesión de 2008-2009 fue más profunda que la de 2000-2002 puesto que la pérdida de empleos fue alrededor de cuatro veces más. Lo mismo puede decirse en cuanto a su severidad, ya que esta pérdida se presentó a una velocidad mayor en términos de duración del descenso. Otro hallazgo interesante es el hecho de que la capacidad potencial para la generación de puestos de trabajo por parte de esta economía ha logrado mantenerse ya que la pendiente que se observa en el filtro HP no cambió de signo. Si bien se ha debilitado, ha logrado para 2010:4 recuperarse en poco tiempo, restableciendo el nivel alcanzado por el pico 2009:6 de su último ciclo.

Esto refleja la fortaleza de una economía con estructuras productivas más sólidas que le permiten volver rápidamente a la utilización óptima de sus recursos productivos. Además, en el gráfico es posible apreciar que el comportamiento del empleo observado está muy cerca del potencial establecido por las condiciones de uso que realiza la economía. Esta característica es muy importante debido a que la estabilidad es un determinante importante para un crecimiento económico sostenido.

En relación con los ciclos económicos que muestra la economía sonorense durante el lapso 1997.7-2010.4 (gráfica segunda inferior), se aprecian dos ciclos de crecimiento: el primero corresponde al lapso 1997.7-2003.8 y el segundo al periodo 2003.9-2009.7. La duración del primer ciclo económico es de 74 meses y la del segundo de 71 meses. Los descensos asociados a estos dos ciclos se presentan durante el lapso 2000.10-2003.8 para el primer ciclo y 2008.4-2009.7 para el segundo, con una duración de 34 y 13 meses respectivamente.

La recesión de 2001-2003 generó una pérdida de 10011 empleos de la economía en su conjunto. La industria de la construcción se debilitó más en este rubro ya que perdió 4366 empleos, seguida de la industria de la transformación con 3147 empleos y el sector agropecuario (agricultura, ganade- 
ría, silvicultura, caza y pesca) con 2085 empleos. La recesión de 2008-2009 tuvo efectos más severos ya que la economía perdió 30703 empleos. Esta cifra significó tres veces más de los empleos perdidos en la recesión anterior. En esta ocasión el sector que más contribuyó a esta pérdida fue la industria de la construcción con 23647 empleos, seguida por la construcción con 4840 y el comercio con 3757 . Al igual que en el resto de los estados fronterizos, la recesión de 2008-2009 fue más profunda y severa que la del primer ciclo debido al mayor número de empleos perdidos y la velocidad en que esta caída ocurrió. Pero el estado de Sonora tuvo la particularidad de que la caída en 2008-2009 no fue tan pronunciada como en las otras entidades.

Lo anterior es un hallazgo interesante debido a que esta economía, sin ser la más importante en el norte de México, logró mantener sus niveles de empleos muy por encima del pico alcanzado por el ciclo anterior y en 2010.4 está cerca de restablecer el pico 2008.4 alcanzado por el último ciclo. Además, el cambio de pendiente que muestra el filtro HP revela que la economía no ha perdido capacidad para generar empleos.

En el diagrama se observa que la economía de Tamaulipas (tercer gráfico superior) muestra dos ciclos económicos durante el lapso 1997.7-2010.4. El primero se encuentra en el periodo 1997.7-2003.8 con una duración de 74 meses, mientras el segundo se fecha durante el lapso 2003.9-2009.6 con una duración de 70 meses.

Los descensos asociados a estos dos ciclos se ubican en el lapso 2000.112003.8 para el primero y 2008.6-2009.6 para el segundo. La duración de estos episodios fue de 34 meses para el primero y 12 para el segundo. La recesión de 20001-2003 generó una pérdida de 48555 plazas de trabajo para la economía en su conjunto. Las industrias de la transformación y de la construcción contribuyeron en mayor medida a esta pérdida debido a que despidieron a 35279 y 15375 trabajadores respectivamente, seguidos por el sector de comunicaciones y transportes con 1360 trabajadores. Por su parte, la recesión de 2008-2009 se tradujo en una reducción de 53970 empleados. La industria de la transformación produjo el despido de 39658 personas, la de la construcción 11586, transportes y comunicaciones 2580 , y comercio y servicios para empresas, personas y el hogar 1803 y 1274 despidos, respectivamente.

La recesión de 2008-2009 fue ligeramente más profunda si se analiza la cantidad de empleos perdidos. No obstante, tuvo mayor severidad puesto 
que ocurrió en un número de meses casi tres veces menor a la presentada en 2001-2003. En esta entidad, el patrón de las dos recesiones observadas permite apreciar que ambas generaron casi la misma pérdida de bienestar si se mide a través del indicador de empleo. En lo que respecta a la caída en el nivel de empleos durante la última recesión, ésta descendió al nivel alcanzado por el pico (2000.11) del ciclo anterior, empero, a partir de entonces se ha observado una recuperación en esta variable. Por su parte, si bien la pendiente del filtro HP cambió a una forma descendente, la recuperación de empleos observada permite vislumbrar que la economía tiende a la utilización óptima de los recursos productivos, además, ha mostrado ser una economía estable como se desprende del empleo observado y su potencial medido por el filtro HP.

El caso de Chihuahua (tercer gráfico parte inferior) es especial ya que siguiendo los resultados reportados por el coeficiente de correlación encontramos que el empleo de esta economía tiene un bajo grado de asociación con el empleo nacional. Éste es un hecho singular en el sentido de que esta economía es la segunda más importante dentro de la frontera norte en cuanto a contribución al PIB nacional y la tercera más importante dentro de la industria manufacturera. Sin embargo, a nivel de empleo la economía muestra algunas características distintas a las mostradas por el resto de las economías de la frontera norte.

Los ciclos de crecimiento de la economía de Chihuahua se encuentran para el lapso 1997.7-2003.5 y 2003.4-2009.6. El primer ciclo tiene una duración de 71 meses y el segundo de 72. La duración del primer ascenso fue de 40 meses y la del segundo de 57. Los periodos de descenso se encuentran en 2000.10-2003.05 para el primer ciclo y 2008.3-2009.6 para el segundo. El primer descenso tiene una duración de 31 meses y el segundo de 15 . Si se observan las fechas de estas recesiones, se encontrará que guardan correspondencia con las establecidas para el resto de las economías de la frontera norte.

La recesión de 2001-2003 muestra una drástica caída en la generación de empleos, equivalente a 122012 de la economía en su conjunto. La contracción más importante se presenta en la industria de la transformación con 108720 despidos, seguida por servicios para empresas, personas y el hogar con la pérdida de 7631 empleos, la industria de la construcción con 4044 empleos y el sector agropecuario con 2349 empleos. Por su parte, la recesión de 2008-2009 muestra una caída similar a la mostrada en el primer 
ciclo con la pérdida de 102117 empleos. Nuevamente, la industria de la transformación contribuye en mayor medida a esta caída con la pérdida de 86387 empleos, seguida por la industria de la construcción y servicios para empresas, personas y el hogar con 7798 y 5575 empleos respectivamente. Un hecho interesante que revelan los datos anteriores es que esta entidad es la única que mostró mayor profundidad en la primera recesión debido a que la pérdida de empleos fue mayor en relación con la de 2008-2009. Sin embargo, ambas recesiones son demasiado profundas y ésta es una característica que distingue al estado de Chihuahua del resto de las entidades. En relación con la severidad de las mismas, se encuentra que la velocidad de la última fue mayor, por lo que, se puede concluir, la recesión de 2008-2009 fue más severa que la de 2001-2003.

Otro hallazgo interesante que debe señalarse es que la expansión del segundo ciclo no consiguió restablecer el empleo alcanzado por el pico (2000.11) del ciclo anterior y la recesión 2008-2009 llevó los niveles de empleo por debajo de la recesión de 2001-2003. Si hubiera oportunidad de utilizar un periodo de tiempo más extenso para observar la dinámica del crecimiento de esta economía, se vería que Chihuahua vive un ciclo depresivo muy extendido, desde 2001 al 2009, en una situación propia de las crisis de los modelos de desarrollo, en la que a lo largo de más de una década, la tendencia de crecimiento no ha sido capaz de regresar a sus niveles de crecimiento. Por último, si se observa el filtro HP se evidencian dos cúspides, lo que expresa la inestabilidad de esta economía para generar empleos y la imposibilidad de regresar a una senda de crecimiento. Éste es un hallazgo vislumbrado en otros estudios para la industria maquiladora de exportación. Se encuentra que en Chihuahua esta actividad muestra una planta laboral en descenso y no ha logrado restablecer los niveles alcanzados por la fase precedente a la crisis de 2001-2003 (Díaz, 2009:39).

Este resultado es interesante pues revela la falta de dinamismo de la economía chihuahuense. La capacidad potencial para generar empleos está en pleno descenso, lo cual refleja que la economía está muy lejos de utilizar óptimamente sus recursos productivos. Esta evidencia debe ser empleada para la toma de decisiones de política económica que permitan revertir las tendencias generales mostradas por los niveles de ocupación.

En resumen, estos resultados agregados se presentan en el cuadro 5. A partir de 1997, en los estados del norte se identifican dos ciclos económicos: 
la oscilación considerada de pico a pico, que delinea un movimiento de descenso -es decir, una recesión-, y el ascenso o recuperación.

El auge que se aprecia en 1997 es resultado de un ciclo más largo que inicia con la recuperación económica luego de la crisis económica de 1995 y ya en la era del TLCAN. Este impulso tuvo diferente impacto en la región de la frontera norte. La duración promedio para estos estados fue de 40.2 meses, siendo Baja California y Coahuila los estados donde el ascenso fue más duradero, pero la expansión fue frenada por la crisis económica internacional asociada con los eventos del 11 de septiembre de 2001. La recesión desatada a principios de la década del 2000 tiene una duración mayor a los 32 meses con excepción del estado de Nuevo León, que inicia su recuperación un año antes que el resto de las entidades. Esta primera crisis, que podemos ubicar entre 2001 y 2003, tiene elevados costos en términos de destrucción de empleos, en especial en el sector manufacturero, aunque también en la industria de la construcción, y con excepción de Chihuahua, resultó más severa que la crisis que sobrevendría después, en 2009.

\section{Cuadro 5.}

Ciclos económicos en los estados de la frontera norte, 1997:7-2010:4

\begin{tabular}{|l|c|c|c|c|}
\hline $\begin{array}{c}\text { Entidad } \\
\text { federativa }\end{array}$ & $\begin{array}{c}\text { Ciclos } \\
\text { económicos }\end{array}$ & $\begin{array}{c}\text { Duración } \\
\text { ascenso en } \\
\text { meses }\end{array}$ & $\begin{array}{c}\text { Duración } \\
\text { descenso } \\
\text { (recesión) } \\
\text { en meses }\end{array}$ & $\begin{array}{c}\text { Empleos } \\
\text { perdidos }\end{array}$ \\
\hline Baja California & $1997: 7-2003: 8$ & 42 & 32 & 42311 \\
\hline & $2003: 9-2009: 6$ & 51 & 15 & 67331 \\
\hline Sonora & $1997: 7-2003: 8$ & 40 & 34 & 10011 \\
\hline & $2003: 9-2009: 7$ & 58 & 13 & 30703 \\
\hline Chihuahua & $1997: 7-2003: 5$ & 40 & 31 & 122012 \\
\hline & $2003: 4-2009: 6$ & 57 & 15 & 102117 \\
\hline Coahuila & $1997: 7-2003: 8$ & 41 & 33 & 37344 \\
\hline & $2003: 9-2009: 5$ & 57 & 11 & 165073 \\
\hline Nuevo León & $1997: 7-2002: 3$ & 38 & 18 & 63434 \\
\hline & $2002: 4-2009: 7$ & 61 & 34 & 48555 \\
\hline Tamaulipas & $1997: 7-2003: 8$ & 40 & 12 & 53970 \\
\hline & $2003: 9-2009: 6$ & 58 & 12 & \\
\hline
\end{tabular}

Fuente: Elaboración propia con base en datos del Imss. 
El costo social de la recesión de 2009, sin embargo, fue consecuencia no sólo de la mayor intensidad que tuvo este episodio recesivo, sino también de la mayor profundidad que alcanzó dicha recesión.

El segundo ciclo económico de los estados de la frontera inicia a mediados y finales del 2003 y fue más extenso en longitud (en promedio se prolongó por 57 meses) ${ }^{18}$ pero menor en profundidad. En este caso, los estados en los que la recuperación fue más extensa fueron Sonora, Nuevo León y Tamaulipas. El panorama general que describe el ciclo económico en la frontera es el de una rápida y prolongada recuperación económica en la segunda mitad de los noventa (40 meses en promedio) que culmina en una recesión extensa (30 meses en recesión) pero de profundidad moderada. En el segundo ciclo la recuperación es más extensa (57 meses) pero de alcance moderado -de hecho, en estados como Chihuahua y Baja California en esta segunda fase de recuperación la actividad económica no retorna al nivel máximo alcanzado en el primer ciclo-. Puede parecer controversial llamar "recuperación económica" al periodo 2004-2007 en las economías fronterizas, pero en lo que sí hay certeza es que en la fase descendente de este ciclo la recesión tuvo una extensión corta pero una profundidad mayor (13 meses en promedio, menos de la mitad de la anterior crisis).

La característica común de ambas recesiones es que no son periodos de ajuste en los que se corrijan los fallos de la estructura económica o que lleven a una modificación de la estrategia económica; quizá eso explique el carácter moderado de la recuperación en 2004-2007. En la primera recesión, un repunte de la demanda de exportaciones y mayores dosis de inversión extranjera se combinaron para impulsar el crecimiento de la economía. La pregunta es si la receta funcionará para la misma enfermedad en un contexto diferente: la economía de la frontera en 2011. De esto dependerá la velocidad que alcance la recuperación y la magnitud del siguiente óptimo de la tendencia de crecimiento.

18 Si consideramos que la recuperación de la crisis de 1995 inició en enero de 1996, y suponiendo que los estados de la frontera habrían mantenido crecimiento en el empleo desde entonces, la recuperación contada a partir de ese mes habría sido de 59 meses, muy cerca de lo que duró la recuperación en el segundo ciclo económico (57 meses). 
Siguiendo con el análisis de la tendencia y el ciclo de crecimiento de las economías de la frontera -y considerando que dependiendo de la estacionalidad de las series, si un shock exógeno saca a la economía de su tendencia será más fácil que ésta regrese a la senda del crecimiento, atribuida a Cochran (1988)-, podemos esperar diferentes resultados del pronóstico de crecimiento económico de estos estados. De acuerdo con esta definición, se estima que Nuevo León es la entidad con mayores posibilidades de retornar al nivel de crecimiento del pasado inmediato, y en segundo término Sonora y Tamaulipas. La economía de Coahuila se encuentra en un tercer nivel de probabilidad de regresar a la senda de crecimiento en un plazo razonable, mientras que, por la tendencia de crecimiento que han seguido en los últimos trece años, Baja California y Chihuahua parecieran tener menores posibilidades de alcanzar los niveles de desempeño logrado, por ejemplo, en la segunda mitad de la década de los noventa. Esto podría ser considerado un resultado del agotamiento del efecto TLCAN.

De esta combinación de resultados podemos concluir que el pronóstico de crecimiento depende crucialmente de la diversificación de la estructura productiva de los estados, en donde tomará más tiempo la recuperación económica de estados que sólo cuentan con estructuras orientadas hacia las exportaciones y dependientes de inversiones extranjeras que la de otras economías en las que también existe este núcleo exógeno pero vinculado a un núcleo endógeno que integra servicios, agroexportaciones y exportaciones industriales de base nacional o de firmas multinacionales productoras de bienes finales.

\section{Modelos VAR para la determinación de comovimientos de la dinámica del empleo regional y nacional}

Los modelos de vectores autorregresivos (VAR) son actualmente considerados como los más adecuados para estudiar las relaciones dinámicas entre las diferentes variables económicas. Éstos han sido utilizados para determinar el grado de comovimiento que expresan las economías regionales con las nacionales. Específicamente, Sherwood-Call (1988) observa la interrelación de los ciclos regionales con los ciclos de escala nacional para la economía de Estados Unidos. Esta estrategia es utilizada en esta investigación para observar el grado de interrelación que muestra el nivel de empleo de las economías 
estatales de la frontera norte con la dinámica del empleo en México. Específicamente, se ha construido una medida de fuerza de cohesión denominada como LINk por Sherwood-Call a través del análisis de descomposición de varianza.

Esta medida de descomposición de varianza tiene la utilidad de pronosticar los efectos que tiene el desempeño de la economía nacional sobre las regionales para, de este modo, discernir cuál de estas últimas muestra una estrecha relación con el comportamiento del país respecto de aquellas que muestran una débil relación. Para llevar a cabo la estimación de los comovimientos se construyeron seis modelos VAR de dos variables para cada una de las economías estatales de la frontera norte. Así, el empleo nacional siempre está presente en cada uno de los modelos y el empleo regional está en función de la economía en cuestión para la cual se construye el var.

En cada caso, se tomó la diferencia del logaritmo del empleo nacional y regional. La información que se utiliza es la misma que empleamos en el apartado anterior para identificar los ciclos particulares de cada economía estatal. Es información proveniente del Imss para los trabajadores permanentes eventuales y urbanos durante el lapso 1997.7-2010.4. Una práctica común para llevar a cabo la estimación de modelos VAR es que las variables que se utilizan para construir el modelo sean estacionarias. Si se utilizan variables no estacionarias, los efectos de un choque transitorio tendrán efectos permanentes, provocando así resultados espurios.

En la práctica econométrica se ha observado que la mayoría de las series económicas son no estacionarias o tienen raíz unitaria. Éstas, en su mayoría, están expuestas a choques que representan cambios no anticipados. Es por eso que los choques transitorios se traducen en efectos permanentes y causan que las series muestren raíz unitaria. La conversión de una serie no estacionaria a una serie estacionaria se da a través de una transformación a diferencia de la variable. Esto es debido a que la mayoría de las series económicas están integradas de orden 1, lo cual suele solucionarse al aplicar la primera diferencia a la variable. En este apartado diferenciamos el logaritmo del empleo nacional y regional para hacerlas estacionarias. En específico, aplicamos la prueba Dickey-Fuller aumentada para observar si las series tienen raíz unitaria. 


\section{Cuadro 6. Pruebas de raíz unitaria}

\begin{tabular}{|c|c|c|c|c|}
\hline \multirow[b]{2}{*}{ Variable } & \multirow[b]{2}{*}{ Especificación } & \multicolumn{2}{|c|}{ Ho: Raíz unitaria } & \multirow{2}{*}{$\begin{array}{c}\text { Orden de } \\
\text { integración }\end{array}$} \\
\hline & & ADF & Decisión & \\
\hline$\Delta \mathrm{E}$ nac. & $\sin c y t d$ & -2.44 & $\mathrm{R}$ & $\mathrm{I}(0)$ \\
\hline$\Delta \mathrm{Ebc}$ & $\sin c y t d$ & -2.93 & $\mathrm{R}$ & $\mathrm{I}(0)$ \\
\hline$\Delta \mathrm{E}$ nac. & $\sin c y t d$ & -2.47 & $\mathrm{R}$ & $\mathrm{I}(0)$ \\
\hline$\Delta$ Ech & $\sin c y t d$ & -2.53 & $\mathrm{R}$ & $\mathrm{I}(0)$ \\
\hline$\Delta \mathrm{E}$ nac. & $\sin c y t d$ & -2.46 & $\mathrm{R}$ & $\mathrm{I}(0)$ \\
\hline$\Delta$ Ecoah & $\sin c y t d$ & -3.63 & $\mathrm{R}$ & $\mathrm{I}(0)$ \\
\hline$\Delta \mathrm{E}$ nac. & $\sin c y t d$ & -2.5 & $\mathrm{R}$ & $\mathrm{I}(0)$ \\
\hline$\Delta \mathrm{Enl}$ & $\sin c y t d$ & -2.3 & $\mathrm{R}$ & $\mathrm{I}(0)$ \\
\hline$\Delta \mathrm{E}$ nac. & $\sin c y t d$ & -2.46 & $\mathrm{R}$ & $\mathrm{I}(0)$ \\
\hline$\Delta$ Eson & $\sin c y t d$ & -2.96 & $\mathrm{R}$ & $\mathrm{I}(0)$ \\
\hline$\Delta \mathrm{E}$ nac. & $\sin c y t d$ & -2.47 & $\mathrm{R}$ & $\mathrm{I}(0)$ \\
\hline$\Delta$ Etam & $\sin c y t d$ & -2.35 & $\mathrm{R}$ & $\mathrm{I}(0)$ \\
\hline
\end{tabular}

Fuente: Elaboración propia.

Los resultados encontrados para las series en niveles muestran que tanto el empleo nacional como el regional tienen raíz unitaria. Es por ello que se realizó la transformación de las series en diferencias, con lo cual se consigue que éstas sean integradas de orden cero y estacionarias. Una vez que las series son estacionarias es posible realizar la especificación del modelo var que se construirá para cada una de las entidades federativas de la frontera norte de México. El modelo tiene las siguientes características:

$$
\begin{aligned}
& \Delta E_{M e x-\operatorname{Reg}, t}=\alpha_{1}+\sum_{i=1}^{p} \beta_{i 1} \Delta E_{M e x-\operatorname{Reg}, t-i}+\sum_{i=1}^{q} \eta_{i 1} \Delta E_{\operatorname{Reg}, t-i}+e_{M e x-\operatorname{Reg}, t} \\
& \Delta E_{\text {Reg, } t}=\left(\alpha_{2}+\beta_{02} \alpha_{1}\right)+\sum_{i=1}^{p}\left(\beta_{i 2}+\beta_{02} \beta_{i 1}\right) \Delta E_{M e x-\operatorname{Reg}, t-i} \\
& +\sum_{i=1}^{q}\left(\eta_{i 2}+\beta_{02} \eta_{i 1}\right) \Delta E_{R e g, t-i}+\beta_{02} e_{M e x-\operatorname{Reg}+} e_{R e g, t}
\end{aligned}
$$

Este sistema de ecuaciones muestra que todas las variables son endógenas, de modo tal, que la variación del empleo regional y nacional está explicado por su misma historia en $t$ periodos atrás y por el error estocástico que afecta a toda la nación y la región. Con base en lo anterior, es necesario 
establecer el orden de rezago para las variables endógenas dentro del sistema de ecuaciones. Los criterios óptimos más usados para la elección del orden del rezago son el criterio de información de Akaike (AIC), el criterio de información de Schwarz y el criterio de información de Hannan-Quinn.

Los resultados obtenidos por medio de estos criterios señalan que los rezagos óptimos para construir los modelos VAR de las entidades federativas de la frontera norte de México son de tres retardos para todos los casos y por todos los criterios (véanse los resultados en el Anexo 1). Por otra parte, es necesario asegurarnos de que los modelos VAR construidos sean estables. Si los efectos de un choque sobre las diferentes variables del sistema se van diluyendo con el paso del tiempo, entonces el VAR construido es estable; si, por el contrario, los choques son persistentes, el modelo es inestable.

Los valores Eigen, también conocidos como raíces características, establecen la condición necesaria y suficiente para que los modelos VAR construidos sean estables. El criterio es que todos los valores Eigen de la matriz de parámetros asociados a las variables endógenas retardadas o a las soluciones del polinomio característico autorregresivo tengan módulo menor a uno. Por tanto, en términos del polinomio autorregresivo, la condición de estabilidad es que todas sus raíces invertidas tengan un valor inferior a uno y residir dentro del círculo unitario. Los resultados de este criterio demuestran que todos los modelos var construidos son estables y estacionarios. En el anexo 2 se reportan los resultados.

Por tanto, para los seis sistemas de ecuaciones especificados en un modelo VAR para las entidades de la frontera norte, se realizaron las pruebas de raíz unitaria para cumplir con la estacionariedad de las series y criterios de rezagos óptimos y estabilidad para asegurarse de que los residuos sean ruido blanco. Con ello puede decirse que los sistemas de ecuaciones del VAR son estables y estacionarios. Una vez bien establecidas las características que deben contener los modelos VAR, la evidencia empírica que éstos producen consiste esencialmente en funciones de impulso-respuesta y descomposición de varianza. Particularmente, esta última es la que interesa ya que permite observar en qué medida el comportamiento de una variable se encuentra determinada por choques inesperados en alguna otra variable. En este caso, se busca establecer qué tanto el empleo regional es explicado por variaciones del empleo nacional, de tal forma que pueda revelar el grado de comovimiento de ambas variables y con ello establecer la correspondencia de sus ciclos económicos medidos por el empleo. 
Ésta es una medida de fuerza de cohesión denominada anteriormente como LINK y permite capturar el grado de comovimiento entre el ciclo de escala nacional y subnacional. Los resultados de esta medida toman valores entre 0 y 1 ; si el valor es cercano a 1, existe un fuerte vínculo de lo que sucede en términos de empleo regional y nacional; si el valor es cercano a cero, el vínculo entre estas variables es débil. Los resultados se presentan a continuación.

Cuadro 7. Análisis de descomposición de varianza para el empleo regional 1997.7-2010.4

\begin{tabular}{|l|c|c|c|}
\hline \multicolumn{1}{|c|}{ Estado } & Periodo (1) & Periodo (36) & Núm. de retardos \\
\hline Baja California & 57.07 & 57.35 & 3 \\
\hline Chihuahua & 39.05 & 21.69 & 3 \\
\hline Coahuila & 45.61 & 47.42 & 3 \\
\hline Nuevo León & 62.13 & 69.93 & 3 \\
\hline Sonora & 57.10 & 62.51 & 3 \\
\hline Tamaulipas & 59.94 & 68.11 & 3 \\
\hline
\end{tabular}

Fuente: Elaboración propia.

Con base en estos resultados, el porcentaje de variación debido a cambios en el empleo nacional en Nuevo León es de $62 \%$ en el corto plazo y $69 \%$ para un periodo de 36 meses adelante. Para Tamaulipas es de $59 \%$ en el corto plazo y $68 \%$ a 36 meses adelante. Para Sonora es de $57 \%$ para el corto plazo y $62 \% 36$ meses adelante respectivamente. Para Baja California, el porcentaje de variación debido a cambios del empleo nacional es de $57 \%$ en el corto plazo y 36 meses adelante. Para Coahuila, el porcentaje de variación es más débil, con 45 y $47 \%$ del corto plazo y 36 meses adelante. Por último, Chihuahua es de $39 \%$ en el corto plazo y disminuye a $21 \% 36$ meses adelante.

El cuadro 7 revela que a través del análisis de descomposición de varianza, la economía estatal más integrada con la economía nacional es la de Nuevo León, lo cual es un rasgo característico dado el tamaño y la importancia de esta entidad, seguida por Tamaulipas, Sonora y Baja California. Por su parte, los estados de Coahuila y Chihuahua muestran una interrelación más débil con la economía nacional, sobre todo el último. Éste es un hecho manifestado en el análisis de los ciclos particulares y sugiere que estas eco- 
nomías pueden estar vinculadas a una dinámica regional distinta -en específico, a la del estado de Texas.

Lo anterior muestra que la dinámica de corto plazo en las entidades de la frontera norte tiene un distinto grado de comovimiento. Mientras algunas entidades muestran una estrecha relación con la economía nacional, otras muestran una relación no tan fuerte. Esto cobra sentido si se considera que las actividades agregadas de los espacios subnacionales en el norte de México pueden estar ligadas a una dinámica regional distinta, puesto que existen cualidades geográficas que pueden insertarlas en una dinámica diferente de la nacional. Otras, por su tamaño, por su importancia y por la capacidad de sus estructuras productivas, pueden ser más resistentes a choques externos a la región. Si bien las evidencias empíricas que de aquí se desprenden no son suficientes para establecer la existencia de ciclos económicos propios de las regiones, sí revela que la manifestación regional de los ciclos económicos es distinta y se expresa en los distintos grados de comovimientos. Esto último nos lleva a la necesidad de seguir documentando los hechos característicos de los ciclos económicos a escala subnacional para establecer su existencia.

\section{Conclusiones}

En este estudio se ha analizado el ciclo económico en los estados de la frontera norte de México. Ante la falta de un indicador de alta frecuencia para la producción agregada por entidad federativa, fue necesario elegir una variable que aproximara el ciclo económico, para lo cual se utilizó un indicador de empleo: las cifras de trabajadores asegurados permanentes y eventuales del IMSS.

En México, el estudio de los ciclos económicos es escaso y para el caso de los ciclos económicos regionales lo es más aún. El interés particular del presente trabajo era conocer la posible existencia de ciclos regionales que permitan reflejar la dinámica económica en un espacio geográfico subnacional, en específico, la frontera norte de México.

Conforme a la tendencia seguida en la literatura sobre los ciclos regionales, en la presente investigación se estudió el ciclo económico en forma dual: en la primera parte se identificaron los ciclos particulares en cada entidad federativa, analizando las fluctuaciones alrededor de una tendencia que muestra los ciclos de crecimiento y la capacidad potencial de crecimiento 
o los ascensos y descensos del indicador cíclico en términos absolutos, lo que se ha denominado como los ciclos clásicos. En una segunda perspectiva, siguiendo a Sherwood-Call (1988:16), se estimó el grado de comovimiento del ciclo nacional y regional para esclarecer la vinculación de las economías de la frontera norte con la economía del país, a diferencia de muchos de los estudios sobre economía regional que postulan la influencia del ciclo económico de Estados Unidos -o de la economía del sur de ese país- sobre las economías de los estados de la frontera mexicana.

La teoría de los ciclos económicos reales establece que una varianza mayor del componente secular en relación con el componente cíclico en una serie de tiempo sugiere que los disturbios reales (no monetarios) son probablemente una fuente más importante de las fluctuaciones en el producto que los disturbios monetarios (Nelson y Plosser, 1982:155). Asimismo, se advierte que las fluctuaciones en el producto se deben más a los cambios en el residuo de Solow -que es la medida de las innovaciones o perturbaciones tecnológicas, de carácter más permanente o de largo plazo- que a factores de corto plazo.

Este enfoque permitió identificar dos recesiones económicas en los estados de la frontera, en 2001-2003 y en 2008-2009, y los dos periodos de recuperación asociados a éstas. Considerando la estacionariedad de las series de tiempo, podemos concluir que en la siguiente recuperación económica, Nuevo León es la entidad con mayores posibilidades de retornar al nivel de crecimiento del pasado inmediato, seguido por Sonora y Tamaulipas. Coahuila parece exhibir menor probabilidad de regresar a la senda de crecimiento en corto plazo, en tanto que Baja California y Chihuahua cuentan con menores posibilidades de alcanzar los niveles de desempeño del pasado. Esto pareciera confirmar cierta evidencia a favor de que el efecto TLCAN se ha extinguido.

De esta combinación de resultados podemos concluir que el pronóstico de crecimiento depende crucialmente de la diversificación de la estructura productiva de los estados.

Sobre el movimiento sincronizado de las economías del norte con el país en su conjunto, el estudio sugiere que las economías de los estados del norte se desplazan en forma sincrónica con la economía de México, a excepción quizá de la economía del estado de Chihuahua, lo cual a su vez sugiere que en la presente etapa de globalización las economías de ambos países han también sincronizado sus ciclos económicos. Esto explica- 
ría el grado de comovimiento de las economías de Baja California, Sonora, Coahuila, Nuevo León y Tamaulipas con la economía de México. En esta visión, si estos estados contribuyen con más de una cuarta parte de la economía del país y se han vinculado (vía las manufacturas de ensamble y el sector automotriz) al comercio internacional con Estados Unido, -de forma que el ciclo estadounidense influye o determina el curso de la economía regional en los estados de la frontera mexicana, como sugieren algunas investigaciones-, no puede entenderse que la economía del país no se encuentre vinculada también con la economía del país del norte. Más aún cuando desde la década de los noventa, el sector exportador dominado principalmente por firmas multinacionales, se ha extendido a otras regiones de México distantes de la región fronteriza.

En este sentido, la investigación aporta evidencias que apuntan hacia la comprobación de la hipótesis de trabajo de la que partimos inicialmente, esto es, que las entidades federativas con mayor participación en el crecimiento de la economía mexicana están más vinculadas con las fluctuaciones de la economía nacional, mientras que las que tienen una menor participación tienen una débil relación. Asimismo, dada la ubicación geográfica y los mayores flujos de comercio y de inversión por el proceso de integración económica con Norteamérica, los estados de la frontera norte muestren ciclos económicos vinculados con fenómenos asociados a su vez a choques internacionales.

\section{Bibliografía}

Acevedo, E. (2009), PIB potencial y productividad total de los factores, Recesiones y Expansiones en México", Economía Mexicana, Nueva Epoca, 18, 2, segundo semestre, pp. 175-219.

Burns, Arthur y Mitchell Wesley (1946), "Measuring business cycles", Nueva York, National Bureau of Economic researck.

Cochran, John H. (1988), "How big is the random walk in GNP?". Journal of Political Economy, vol. 96, num. 51, pp. 893-920.

Cuadra, Gabriela (2008), "Hechos estilizados del ciclo económico en México", Banco de México, Documentos de investigación, diciembre, pp. 1-54.

Delajara, Marcelo (2010), "Comovimiento y concordancia cíclica del empleo en los Estados Mexicanos", Banco de México, Documento de Investigación, núm. 2010-13, México. Disponible en <http://www.banxico.org.mx/ 
publicaciones-y-discursos / publicaciones / documentos-de-investigacion / banxico/\% 7BC36FB86D-77BD-E8A9-1C16-08D62F09EEC9\%7D.pdf>.

Díaz G., Eliseo (2009). "Crecimiento económico sostenido y productividad regional”,

Boletín del Sistema Nacional Estadístico y de Información Geográfica, núm. 1, México,

Instituto Nacional de Estadística, Geografía e Informática (INEGI), pp. 23-40.

INEGI (2008), "Sistema de indicadores compuestos: coincidente y adelantado", Documento metodológico, México, INEGI.

Erquizio, Oscar (2001), "Recesión 2001 en México", en Vértice Universitario, 2, 11, julio-septiembre, pp. 3-7.

(2003), "Identificación del ciclo económico en Sonora", en Vértice Universitario, 5, 19, Julio-Septiembre, pp. 3-6.

(2006a), "Ciclos económicos en la frontera norte de México", en Vázquez Ruiz y Bocanegra, Desarrollo regional y Local, Universidad de Sonora/Hermosillo, Sonora, México, 2006, pp. 47-76.

(2006b), Ciclos económicos en México, Hermosillo, Sonora, Universidad de Sonora.

(2007a), "Ciclos económicos secto-regionales en México 1980-2006”, Chetumal,/Quintana Roo, 25 de Mayo de 2007.

(2007b), "Identificación de los ciclos económicos en México 1949-2006", en Problemas del Desarrollo, 38, 150, julio-septiembre, pp. 235-250.

y Mario Mendoza (2008), "Crecimiento y ciclos regionales: Antes y después del Tratado de Libre Comercio de América del Norte”, en Vázquez Ruíz (coord.), La economía mexicana antes y después del TLCAN, Hermosillo, Universidad de Sonora, pp. 35-74.

Félix, V., Gustavo y Alejandro Dávila F. (2008). "Apertura comercial y demanda en el crecimiento de las entidades federativas de México”. Comercio Exterior, vol. 58, núm. 4, abril, México.

Instituto Mexicano del Seguro Social (IMSs), Trabajadores permanentes y eventuales urbanos, Cubo de información directiva C.P. Consultado en <http://www.imss. gob.mx/estadisticas/financieras/cubo.htm>.

Keynes, J. M. (1936), La teoría general de la ocupación, el interés y el dinero, Buenos Aires, FCE.

Kydland, F. E. y E. C. Prescott (1982), "Time to build and aggregate fluctuations", Econométrica, vol. 50, núm. 6, noviembre, pp. 1345-70.

(1988), "The workweek of capital and its cyclical implication", Journal of Monetary Economic”, 21, pp. 344-360.

(1990), "The econometrics of the general equilibrium approach to business cycle”, Federal Reserve Bank of Minneapolis, reporte 130, noviembre, pp. 1-26.

Loria, E., M. Ramos y L. de Jesús (2008), "Producto potencial y ciclos económicos en México 1980.1-2006.4”, Estudios Económicos, 23, 1, El Colegio de México, enero-junio, pp. 25-48. 
Lucas, Robert (1977), "Understanding business cycle”, Carnegie-Rochester series on public policy, 5, pp. 7-29.

Mankiw, Gregor (1989), "Real business cycle: a new keynesian perspective", The Journal Economics Perspectiv", 3, 3, verano, pp. 79-90.

Mejía, Pablo (2002), "Ciclos económicos en México”, Documentos de Investigación 68, El Colegio Mexiquense, pp. 2-40.

(2003a), "Fluctuaciones cíclicas de la industria maquiladora en México", Frontera Norte 15, 29, El Colegio de la Frontera Norte, enero-junio, pp. 65-83.

(2003b), "Regularidades empíricas en los ciclos económicos de México: producción, inversión, inflación y balanza comercial”, Economía Mexicana 12, 2, julio-diciembre, pp. 231-274.

NBER (2010), "Statement of NBER Business cycle dating committee on determination of dates turning points in the U.S. economy". Consultado el 23 de mayo de 2010, disponible en: <http://www.nber.org/cycles/main.html>.

Nelson, Charles y Charles Plosser (1982), "Trends and random walk in macroeconomics time series", Journal of Monetary Economics, 10, pp. 140-162.

Romer, David (2006),"Advanced Macroeconomic”, Estados Unidos, McGraw-Hill.

Samuelson, Paul (1939), "Multiplier-accelerator model”, The review of economic statistic, 75-78, Cambridge, Mass., Harvard University.

Schumpeter, Joseph (1912, 1944), "Teoría del desenvolvimiento económico”, México, FCE.

Sherman, Howar (1991), "Endogenous and exogenous cycle theories", en Ciclos económicos: morfología, modelos y teorías, trad. Alfredo Erquizio, Hermosillo Sonora, Universidad de Sonora, pp. 163-225.

Sherwood-Call, Caroline (1988), "Exploring the relationship between national and regional fluctuations", Economic Review, 3, verano, pp. 15-25.

Summers, Lawrence (1986), "Some skeptical observation on real business cycle theory", Federal Reserve Bank of Minneapolis Quarterly, 10, 4, pp. 23-27.

Timbergen, J. (1935), "Suggestions on quantitative business cycle theory", Econometrica, 3, 3, julio, pp. 241-308.

Torres, A. (2000), "Estabilidad de variables nominales y el ciclo económico. El caso de México”, Banco de México, Documento de Investigación, núm. 2000-03.

Zuccardi Huertas, Igor (2002), "Los ciclos económicos regionales en Colombia 1986-2000", Centro de estudios económicos regionales, Banco de la República, 25. Disponible en: <http://ideas.repec.org/s/col/000102.html>.

Artículo recibido el 31 de marzo de 2011 Segunda versión recibida el 31 de octubre de 2011 Artículo aprobado el 12 de noviembre de 2011 


\section{ANEXO 1. Criterios de retardos óptimos}

Orden de criterio de selección de rezagos VAL

Variable endógena DEL_estado_DEL

Variable exógena: $\mathrm{C}$

Muestra: 1997M07 2010M04

Observaciones incluidas: 145

\begin{tabular}{|c|c|c|c|c|c|c|c|}
\hline $\begin{array}{c}\text { Baja } \\
\text { California }\end{array}$ & Lag & $\log L$ & LR & FPE & AIC & SC & $H Q$ \\
\hline & 0 & 1215.538 & NA & $1.84 \mathrm{e}-10$ & -16.73846 & -16.69740 & -16.72178 \\
\hline & 1 & 1231.479 & 31.22141 & $1.56 e-10$ & -16.90316 & -16.77998 & -16.85311 \\
\hline & 2 & 1245.267 & 26.62503 & $1.37 e-10$ & -17.03816 & -16.83287 & -16.95475 \\
\hline & 3 & 1268.179 & $43.61283^{*}$ & $1.05 \mathrm{e}-10^{*}$ & $-17.29903^{*}$ & $-17.01162^{*}$ & $-17.18224^{*}$ \\
\hline & 4 & 1270.620 & 4.578572 & $1.08 \mathrm{e}-10$ & -17.27752 & -16.90799 & -17.12737 \\
\hline & 5 & 1271.618 & 1.844762 & $1.12 \mathrm{e}-10$ & -17.23611 & -16.78447 & -17.05260 \\
\hline & 6 & 1272.674 & 1.921390 & $1.17 e-10$ & -17.19550 & -16.66174 & -16.97861 \\
\hline & 7 & 1277.087 & 7.913524 & $1.16 e-10$ & -17.20120 & -16.58532 & -16.95095 \\
\hline & 8 & 1278.275 & 2.097879 & $1.21 \mathrm{e}-10$ & -17.16242 & -16.46442 & -16.87880 \\
\hline \multirow[t]{10}{*}{ Chihuahua } & Lag & LogL & LR & FPE & AIC & sc & $H Q$ \\
\hline & 0 & 1185.467 & NA & $2.79 \mathrm{e}-10$ & -16.32368 & -16.28263 & -16.30700 \\
\hline & 1 & 1227.024 & 81.39462 & $1.66 e-10$ & -16.84171 & -16.71854 & -16.79166 \\
\hline & 2 & 1241.023 & 27.03138 & $1.45 e-10$ & -16.97962 & -16.77433 & -16.89620 \\
\hline & 3 & 1265.176 & $45.97562^{*}$ & $1.10 \mathrm{e}-10^{*}$ & $-17.25761^{*}$ & $-16.97020^{*}$ & $-17.14082^{*}$ \\
\hline & 4 & 1267.947 & 5.196726 & $1.12 \mathrm{e}-10$ & -17.24064 & -16.87112 & -17.09049 \\
\hline & 5 & 1268.492 & 1.007318 & $1.17 e-10$ & -17.19299 & -16.74135 & -17.00947 \\
\hline & 6 & 1269.912 & 2.585216 & $1.21 \mathrm{e}-10$ & -17.15740 & -16.62364 & -16.94052 \\
\hline & 7 & 1273.801 & 6.973923 & $1.22 \mathrm{e}-10$ & -17.15588 & -16.54000 & -16.90562 \\
\hline & 8 & 1276.883 & 5.440555 & $1.23 e-10$ & -17.14321 & -16.44521 & -16.85959 \\
\hline \multirow[t]{10}{*}{ Coahuila } & Lag & LogL & LR & FPE & AIC & SC & $H Q$ \\
\hline & 0 & 1213.566 & NA & $1.89 \mathrm{e}-10$ & -16.71125 & -16.67020 & -16.69457 \\
\hline & 1 & 1245.315 & 62.18377 & $1.29 \mathrm{e}-10$ & -17.09400 & -16.97082 & -17.04395 \\
\hline & 2 & 1256.608 & 21.80787 & $1.17 e-10$ & -17.19459 & -16.98930 & -17.11118 \\
\hline & 3 & 1275.928 & 36.77482 & $9.46 \mathrm{e}-11^{*}$ & $-17.40591^{*}$ & $-17.11850^{*}$ & $-17.28912^{*}$ \\
\hline & 4 & 1278.390 & 4.618599 & $9.66 \mathrm{e}-11$ & -17.38469 & -17.01517 & -17.23454 \\
\hline & 5 & 1280.034 & 3.038693 & $9.98 \mathrm{e}-11$ & -17.35220 & -16.90056 & -17.16868 \\
\hline & 6 & 1285.698 & 10.31262 & $9.76 \mathrm{e}-11$ & -17.37515 & -16.84139 & -17.15827 \\
\hline & 7 & 1291.720 & $10.79635^{*}$ & $9.50 \mathrm{e}-11$ & -17.40303 & -16.78715 & -17.15278 \\
\hline & 8 & 1295.889 & 7.360421 & $9.48 e-11$ & -17.40536 & -16.70737 & -17.12174 \\
\hline
\end{tabular}




\begin{tabular}{|c|c|c|c|c|c|c|c|}
\hline $\begin{array}{l}\text { Nuevo } \\
\text { León }\end{array}$ & Lag & LogL & LR & FPE & AIC & SC & $H Q$ \\
\hline & 0 & 1272.908 & NA & $8.36 e-11$ & -17.52976 & -17.48870 & -17.51308 \\
\hline & 1 & 1286.536 & 26.69211 & $7.32 \mathrm{e}-11$ & -17.66256 & -17.53939 & -17.61251 \\
\hline & 2 & 1304.226 & 34.16021 & $6.06 e-11$ & -17.85139 & -17.64610 & -17.76797 \\
\hline & 3 & 1324.408 & 38.41606 & $4.85 \mathrm{e}-11^{*}$ & $-18.07460^{*}$ & $-17.78719^{*}$ & $-17.95781^{*}$ \\
\hline & 4 & 1328.092 & 6.910184 & $4.87 e-11$ & -18.07023 & -17.70071 & -17.92008 \\
\hline & 5 & 1329.888 & 3.319681 & $5.02 e-11$ & -18.03984 & -17.58819 & -17.85632 \\
\hline & 6 & 1329.999 & 0.202289 & $5.30 e-11$ & -17.98620 & -17.45244 & -17.76931 \\
\hline & 7 & 1336.832 & $12.25221^{*}$ & $5.10 e-11$ & -18.02527 & -17.40939 & -17.77502 \\
\hline & 8 & 1339.130 & 4.056786 & $5.22 e-11$ & -18.00179 & -17.30380 & -17.71817 \\
\hline \multirow[t]{10}{*}{ Sonora } & Lag & LogL & LR & FPE & AIC & SC & $H Q$ \\
\hline & 0 & 1202.352 & NA & $2.21 \mathrm{e}-10$ & -16.55658 & -16.51552 & -16.53990 \\
\hline & 1 & 1213.180 & 21.20783 & $2.01 \mathrm{e}-10$ & -16.65076 & -16.52758 & -16.60071 \\
\hline & 2 & 1227.512 & 27.67619 & $0.75 e-10$ & -16.79327 & -16.58798 & -16.70986 \\
\hline & 3 & 1251.149 & 44.99078 & $1.33 e-10^{*}$ & $-17.06412^{*}$ & $-16.77671^{*}$ & -16.94734 \\
\hline & 4 & 1251.805 & 1.231078 & $1.39 e-10$ & -17.01800 & -16.64847 & -16.86785 \\
\hline & 5 & 1252.112 & 0.567201 & $1.47 e-10$ & -16.96706 & -16.51542 & -16.78354 \\
\hline & 6 & 1252.464 & 0.641650 & $1.54 \mathrm{e}-10$ & -16.91675 & -16.38299 & -16.69986 \\
\hline & 7 & 1259.281 & $12.22288^{*}$ & $1.49 e-10$ & -16.95560 & -16.33972 & -16.70535 \\
\hline & 8 & 1262.887 & 6.349744 & $1.50 \mathrm{e}-10$ & -16.95003 & -16.25204 & -16.66642 \\
\hline \multirow[t]{10}{*}{ Tamaulipas } & Lag & LogL & LR & FPE & AIC & sc & $H Q$ \\
\hline & 0 & 1246.636 & NA & $1.20 \mathrm{e}-10$ & -17.16740 & -17.12634 & -17.15072 \\
\hline & 1 & 1260.600 & 27.34929 & $1.05 e-10$ & -17.30483 & -17.18165 & -17.25478 \\
\hline & 2 & 1280.456 & 38.34312 & $8.41 e-11$ & -17.52353 & -17.31824 & -17.44012 \\
\hline & 3 & 1300.210 & 37.60059 & $6.77 \mathrm{e}-11^{*}$ & $-17.74083^{*}$ & $-17.45342^{*}$ & $-17.62405^{*}$ \\
\hline & 4 & 1303.455 & 6.087642 & $6.84 \mathrm{e}-11$ & -17.73042 & -17.36089 & -17.58027 \\
\hline & 5 & 1304.436 & 1.812390 & $7.13 e-11$ & -17.68877 & -17.23713 & -17.50526 \\
\hline & 6 & 1305.740 & 2.374528 & $7.40 \mathrm{e}-11$ & -17.65159 & -17.11783 & -17.43471 \\
\hline & 7 & 1312.694 & $12.46797^{*}$ & 7.11e-11 & -17.69232 & -17.07645 & -17.44207 \\
\hline & 8 & 1316.321 & 6.405056 & $7.15 \mathrm{e}-11$ & -17.68719 & -16.98920 & -17.40357 \\
\hline
\end{tabular}

- Indicates lag order selected by the criterion

- LR: sequential modified LR test statistic (each test at 5\% level)

- FPE: final prediction error

- $\quad$ AIC: Akaike information criterion

- SC: Schwarz information criterion

- HQ: Hannan-Quinn information criterion 


\section{ANEXO 2. Círculo unitario}

\section{Baja California}

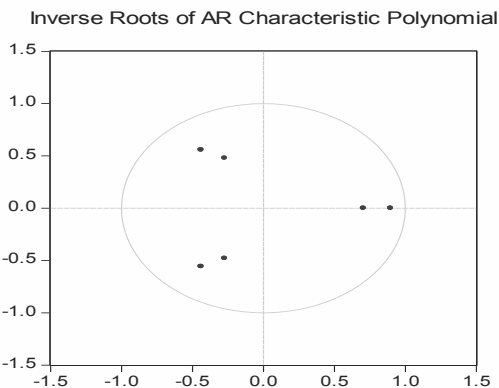

Coahuila

Inverse Roots of AR Characteristic Polynomial

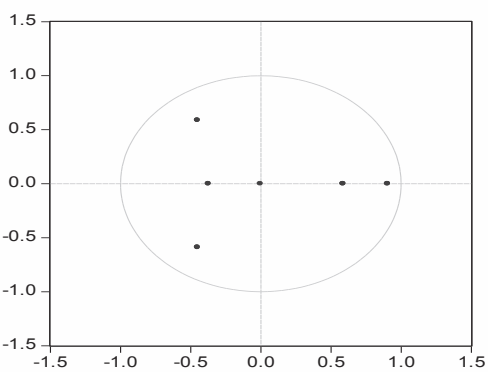

Sonora

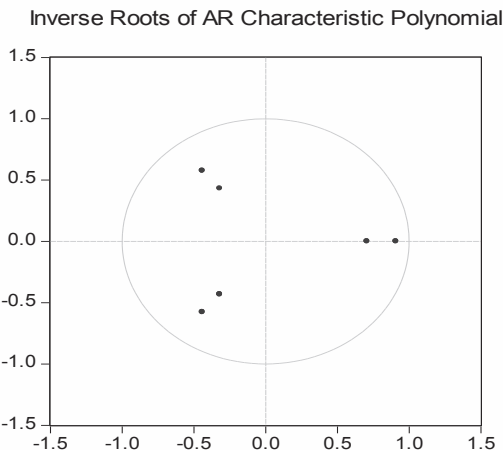

\section{Chihuahua}

Inverse Roots of AR Characteristic Polynomial

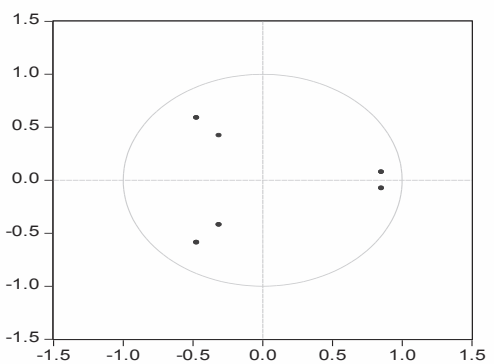

Nuevo León

Inverse Roots of AR Characteristic Polynomial

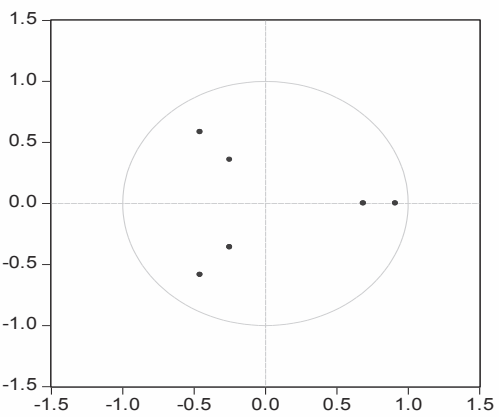

Tamaulipas

Inverse Roots of AR Characteristic Polynomial

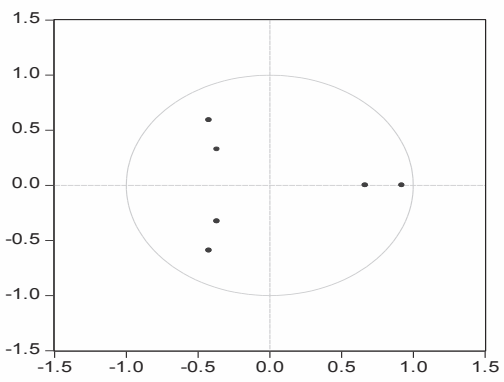

Fuente: Elaboración propia con base en datos del IMss. 